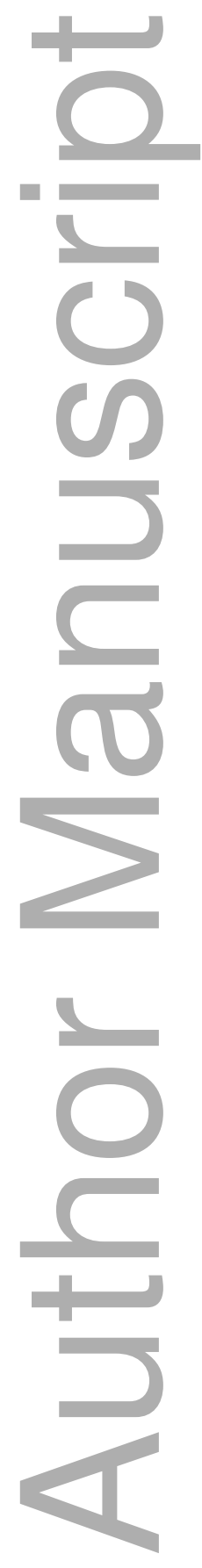

This is the author manuscript accepted for publication and has undergone full peer review but has not been through the copyediting, typesetting, pagination and proofreading process, which may lead to differences between this version and the Version of Record. Please cite this article as doi: $\underline{10.1111 / 1475-4932.12315}$

This article is protected by copyright. All rights reserved 


\section{Designing biosecurity inspection regimes to account for stakeholder incentives: An inspection game - approach}

that reduce intervention and encourage importers to decrease the likelihood of biosecurity risk material being present in consignments. The interaction between a biosecurity regulator and a vertically integrated importer is framed as an inspection game. Our principal focus is a dynamic version of the game, which we use to assess whether regimes based on past compliance can encourage behaviours consistent with the regulatory objective. Our results suggest appropriate candidates for compliance-based inspection regimes are goods where there is access to costeffective "fixed" abatement technologies, and those with high costs associated with being inspected or failing inspection.

JEL Classifications: D82; L51; Q18.

Keywords: biosecurity; inspection game; incentive regulation; continuous sampling plan 


\section{Introduction}

As globalisation results in a larger volume and range of products being traded internationally, managing the spread of invasive species becomes more challenging. Implementing measures to manage biosecurity risks associated with international trade is costly, with the Australian Government spending more than $\$ 600$ million a year on activities related to managing biosecurity and imported food risk (Commonwealth of Australia, 2016). The Australian Government Department of Agriculture and Water Resources (DAWR) is responsible for designing and implementing the rules that govern importing processes and has a range of measures available to it to reduce the risk of entry, establishment and spread of exotic pests and diseases to Australia. These encompass pre-border, border and post-border interventions, with pre-border and border inspections the main options for reducing the likelihood of biosecurity risk material entering the country.

When designing and implementing inspection rules that govern importing processes, biosecurity regulators must balance competing objectives. The primary consideration of Australia's biosecurity system is to preserve Australia's reputation as a producer of reliable clean, green and safe premium products (Commonwealth of Australia, 2015). Australia's highly prized animal and plant health status allows access to lucrative overseas markets and assists the competitiveness of Australia's agricultural exports. A high biosecurity status also supports other aspects of Australia's unique environment, benefiting sectors such as tourism. While preserving a high biosecurity status, the Australian Government must also enable international trade. Trade between countries improves access to higher-quality and/or lower-cost goods for local businesses and consumers, but this may expose Australia to a greater range of pests and diseases.

The leakage of biosecurity risk material into the environment can impose significant costs on the local economy (see Buetre et al. (2013) and Hafi et al. (2015) for some recent estimates for Australia) and environment, and could be irreversible unless eradication is 
deemed technically feasible and cost-beneficial. ${ }^{1}$ Inspection activities and other regulatory requirements set by the DAWR impose direct and/or delay costs on various parties in the supply chain for imported goods. Governments may also dislike imposing regulatory burden on stakeholders, even if they can recover the full costs of their inspection activities from importers. Furthermore, they may consider how these regulatory activities influence the cost and availability of imported products for the Australian public - an aspect often overlooked in assessing biosecurity policies (James and Anderson, 1998). In balancing these competing policy objectives and determining where to direct limited biosecurity resources, the DAWR has been exploring opportunities to better manage biosecurity risks arising from international movements of goods. This involves moving from a "command and control" regulatory framework with mandatory inspection requirements for many types of products, towards a more flexible system, as advocated in previous reviews of Australia's biosecurity system (e.g. Beale et al., 2008), that considers the risks a consignment poses to the biosecurity objective.

In this paper, we explore the potential for a biosecurity regulator to design border inspection rules that account for its own policy trade-offs and the strategic response of regulated entities to these rules. This is akin to an incentive regulation problem, where the biosecurity regulator seeks to design a system of rewards and/or punishments for regulated entities so as to better align those entities' objectives with the government's regulatory objective. Rather than taking a full mechanism design approach, we analyse the interaction between a vertically integrated importer and a biosecurity regulator where the regulator commits to implement a rule from a set of simpler policy rules; this is similar to the treatment in Munford (1981). While more sophisticated and flexible rules, including those that could be adapted via Bayesian updating (e.g. Chun, 2010), could deliver theoretically more optimal policies in this context, such rules may not be

\footnotetext{
${ }^{1}$ For example, eradiction was deemed technically infeasible for Myrtle rust (Urango rangelii) detected in New South Wales in 2010 (Plant Health Australia, 2011), while eradication measures were pursued in response to the banana freckle (Phyllosticta cavendishii) outbreak in the Northern Territory in 2013 (Commonwealth of Australia, 2014).
} 
easily implementable in practice. In particular, they are likely to be difficult to incorporate into existing regulatory and legislative frameworks, and could be challenging for frontline inspection staff to apply and for stakeholders to comprehend. As highlighted by Barr et al. (2013) and others, stakeholders may then behave very differently from the theoretical predictions in such complex decision environments.

The three rules considered in this paper (CSP-1, CSP-2 and CSP-3), which allow the frequency of inspection to depend on the importer's compliance history, are from the continuous sampling plan (CSP) family originally developed in the statistical quality control literature (Dodge, 1943; Dodge and Torrey, 1951). They have the desirable property of being Markovian ${ }^{2}$ in structure and therefore require limited record-keeping to implement. These rules posit that the likelihood of biosecurity risk material being present in a consignment is related to an importer's past compliance, with a lower inspection frequency afforded to entities with a history of bringing in consignments free from biosecurity risk material. ${ }^{3}$ Rules of this nature have been adopted by the DAWR to reduce the level of intervention on imported food products and, more recently, for biosecurity inspections for several "low-risk" plant-based products using the CSP-3 algorithm based on an importer's compliance record.

Our treatment of the biosecurity inspection problem is distinct in the literature, because we analyse the choice of rules by the regulator that affect inspection frequency and the importer's response to these rules from a game-theoretic perspective. Other contributions in the economics literature have focused different aspects of biosecurity management, such as:

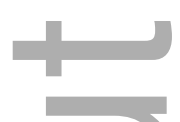

- the complementarity of actions of different firms or producers for reducing biosecu-

\footnotetext{
${ }^{2}$ See the Online Appendix for a more detailed discussion of the theoretical properties of these rules and a brief discussion of alternative rule families discussed in the statistics literature.

${ }^{3}$ The idea is somewhat similar to the two-group audit framework in Friesen (2003), though the CSP rules in themselves do not represent an optimal targeting strategy for enforcement. The other main difference is importers subject to the lower inspection rate only move back to a more intensive intervention schedule if a consignment fails inspection, rather than at random under the audit framework.

${ }^{4}$ As at November 2016, 21 categories of plant-based products, such as dried apricots, green coffee beans and almonds, are part of the DAWR's Compliance-Based Inspection Scheme.
} 
rity risks (e.g. Hennessy, 2008; Kobyashi and Melkonyan, 2011);

- the allocation of inspection effort when the regulator faces capacity constraints (Surkov et al., 2008);

- commercial contracting for farm-level biosecurity management (Abougamos et al., 2012);

- the role of risk aversion and learning dynamics in the adaptive management of invasive species risk (Springborn, 2014); and

- the use of other policy instruments by regulators such as tradable permits (e.g. Horan and Lupi, 2005), technical assistance grants for exporter risk abatement (Fernandez and Sheriff, 2013) or import tariffs (e.g. McAusland and Costello, 2004).

Furthermore, previous investigations of compliance-based protocols in the biosecurity context for Australia (e.g. Robinson et al., 2012) have focused on statistical approaches that do not consider the behavioural response of regulated entities to alternative inspection rules.

Compliance-based protocols, such as those in the CSP family, possess inherent incentive structures to which importers and others in the supply chain may respond. Since stakeholders will respond based on their own motivations, which may or may not be aligned with the government's regulatory objective, understanding the likely behavioural responses to rule changes in itself is important for understanding how effective compliancebased protocols can be as a regulatory tool. Of particular interest from a policy perspective is to understand:

- the circumstances under which compliance-based protocols can be more beneficial for the biosecurity regulator than a mandatory inspection system;

- the types of importers and/or goods most suited to being inspected under compliancebased protocols; 
- how these protocols can be used to encourage importers to act in ways that reduce the likelihood of biosecurity risk material being present in consignments; and

- how specific rule forms and parameters can be chosen ${ }^{5}$ to meet regulatory objectives and encourage supportive stakeholder responses.

An inspection game, as described in Avenhaus et al. (2002), is well-suited to consider the interaction between the biosecurity regulator and an importer. Variants of inspection games have been applied in several settings, including public transport ticketing (e.g. Avenhaus, 2004; Delle Fave et al., 2014), financial auditing (e.g. Besancenot and Vranceanu, 2007; Chou et al., 2012; Krawczyk, 2009), doping in sports (e.g. Berentsen et al., 2008; Kirstein, 2014), customs and smuggling operations (e.g. Avenhaus and Krieger, 2011; Bakır, 2011; Hohzaki, 2011) and environmental management (e.g. Franckx, 2002; Friesen, 2006). The inspection game with inspector leadership is particularly appropriate in our context because the biosecurity regulator can credibly announce the inspection rule to be followed and has the opportunity to commit to a particular strategy. Evidence from administrative data held by the DAWR and discussions with stakeholders ${ }^{6}$ suggest there are two main types of supply chains for imported products, namely:

- vertically integrated relationships, where the importer exclusively (or almost exclusively) sells products from one supplier and effectively operates as that supplier's Australian subsidiary; and

- importers who source products from a large number of independent suppliers, either through an overseas contact who acts like a broker on behalf of multiple suppliers or through direct contact with individual suppliers.

For methodological reasons, we consider a vertically integrated importer model in this paper. This allows a greater focus on the role of the inspection rules without the compli-

\footnotetext{
${ }^{5}$ The game-theoretic and decision-theoretic approaches adopted in this paper augment existing approaches based on statistical criteria (e.g. Hillier, 1964) and allow for a more nuanced approach accounting for scheme costs, consistent with Anscombe (1958).

${ }^{6}$ For a more extensive discussion of findings from CEBRA Project 1304C, see Rossiter et al. (2016).
} 
cation of having to consider how importers might form beliefs about the biosecurity risk material approach rates of alternative suppliers.

To inform discussion of this policy issue, we develop theoretical models that adopt several features of the inspection game with leadership (see e.g. Andreozzi, 2004; Avenhaus et al., 1991), including the assumption that inspection is costly for the inspector - a feature that distinguishes inspection games from monitoring or enforcement games (e.g. Kilgour, 1994; Kirstein, 2014). Our formulation of the inspection problem follows an "acceptzero" approach, where the inspection seeks to establish whether biosecurity risk material is present in or absent from a consignment. ${ }^{7}$

This application includes aspects not normally considered in the standard formulation of an inspection game. We assume the importer chooses inputs to the production process, in the form of fixed technologies and/or variable effort, which affects the probability that a consignment contains biosecurity risk material. In contrast, a more "standard" approach would have the importer making the (deliberate) decision whether or not to comply with biosecurity requirements prescribed by the importing country. Together with the inspector leadership assumption, this formulation allows us to consider pure-strategy equilibria and means the costs of inspection and treatment of contaminated consignments influence the importer's optimal strategy. ${ }^{8}$ Our framework also allows for the inspection process to be imperfect ${ }^{9}$ - an important aspect of practical inspections noted elsewhere in the literature (e.g. Krieger, 2011; Rothenstein and Zamir, 2002).

The remainder of the paper is structured as follows. Section II considers a one-shot

\footnotetext{
${ }^{7}$ An extension of this framework to consider more general compliance tests, such as adhering to a specified standard for seed purity or a residue limit, would require embedding a hypothesis-testing framework akin to inspection games for material accountancy (e.g. Avenhaus et al., 2002) to derive optimal statistically based tests.

${ }^{8}$ Pradiptyo (2007) notes that inspection games with mixed-strategy equilibria can have punishment costs being irrelevant for the inspectee's optimal strategy. In our model, mixed-strategy equilibria only occur on a small subset of the parameter space when particular equality constraints are satisfied - restrictions that are unlikely to be satisfied in practice.

${ }^{9} \mathrm{Jin}$ and Lee (2011), for example, discuss the motivations for inspection errors in a practical setting. While the impact of inspection errors on the CSP-1 algorithm has been investigated by Case et al. (1973), this paper focuses on the behavioural implications of decision-errors by the regulator in the game.
} 
interaction between a biosecurity regulator and vertically integrated importer where the regulator is the leader in the game and is allowed to make decision-errors in inspection. This simplified framework allows analytical solutions and helps provide intuition around the effect of certain model parameters on importer and regulator behaviour through comparative statics. The remainder of the paper considers the more realistic dynamic version of the inspection interaction. In Section III, we introduce the CSP family of rules used as candidate policy rules by the regulator in the dynamic inspection game discussed in Section IV. We conclude in Section V with some observations about the applicability of compliance-based inspection protocols as a strategy for managing biosecurity risks at the border

\section{One-shot biosecurity inspection game}

\section{i Game description}

We first consider the inspection game between the regulator and importer resulting from a one-off import of a homogeneous good. For analytical tractibility, both agents are assumed to be risk-neutral decision-makers.

The importer's objective is to maximise the expected profit from importing this single good. This formulation has the advantage of allowing a "participation" constraint for the importer to be taken into account, since the importer would only import the good if they expected to receive a non-negative return. If the good is brought into the domestic market, the importer receives a guaranteed return of one as a normalisation. ${ }^{10}$

In the one-off importing situtation, the vertically integrated importer chooses how much abatement effort, ${ }^{11} a \geq 0$, to exert in cleaning the consignment. The abatement effort

\footnotetext{
${ }^{10}$ While this abstracts from costs not directly connected to the biosecurity abatement or inspection process, such as transport and storage, the revenue received by the importer can be interpreted as the return net of other costs associated with landing the consignment.

${ }^{11}$ In this context, the distinction between fixed and variable costs is immaterial, so we assume the
} 
costs the importer $a$ (relative to sales revenue) and is linked to the likelihood of a consignment containing biosecurity risk material through the abatement technology function, $\phi(a)$. This function is assumed to be common knowledge, at least once continuously differentiable and satisfies $\phi(\cdot) \in[0,1], \phi^{\prime}(\cdot)<0$ and $\phi^{\prime \prime}(\cdot)>0$ for $a \geq 0$. This means its inverse function is well-defined and the importer's objective function is concave in effort. Note that the regulator does not need to observe the effort exerted by the importer, meaning this formulation takes the moral hazard dimension of the inspection problem into account.

The regulator is concerned with whether the consignment contains biosecurity risk material and the payoffs reflect the inspection outcome. If the regulator inspects the consignment, the importer incurs an additional cost $\omega>0$, reflecting both direct fees charged by the regulator and indirect costs, such as storage and handling charges and delays in getting the product to market. If an inspected consignment contains biosecurity risk material, the importer incurs a rectification (or treatment) cost $\kappa$ in addition to the cost of being inspected.

The biosecurity regulator moves first in the game, choosing the probability of inspection, $\beta \in[0,1]$, and the cost incurred by the importer in having their consignments inspected $(\omega){ }^{12}$ The cost parameter $\omega$ does not enter the regulator's utility function but affects the importer's expected return from their importing activities; a "high" inspection cost may result in the importer being better off (in an expected utility sense) by not importing the good. If the good is not brought into the domestic market, we assume there is no "social loss" incurred by the regulator.

In choosing the probability of inspection, the regulator wants to minimise the expected social cost of intervening in the goods market. The regulator would rather not inspect the importer chooses their effort level.

${ }^{12}$ From a regulatory perspective, $\omega$ should reflect the efficient cost of providing biosecurity inspections plus indirect costs the importer incurs in meeting these requirements. There are also limits around how high inspection fees can be set, most notably from the Agreement on the Application of Sanitary and Phytosanitary Measures (World Trade Organisation, n.d.) and the Australian Government's Cost Recovery Guidelines (Australian Government Department of Finance, 2014). 
consignment, as inspection incurs a cost to society of $\chi>0$ regardless of its outcome. This reflects the opportunity cost of using the government's resources in the inspection process, any costs in delivering the biosecurity system not recovered from the importer and/or the government's implicit preference to avoid regulatory intervention. As a normalisation, choosing not to inspect a "clean" consignment incurs no cost to the regulator. ${ }^{13}$ If the consignment is contaminated but not treated at the border, the regulator internalises the expected social cost $\gamma \gg \chi$ from biosecurity risk material escaping into the domestic market. ${ }^{14}$ The consequences of leakage could include costs associated with having to remedy biosecurity risks that become established, such as those arising from eradication programs, or broader economic costs stemming from loss of export market access or reduced agricultural productivity.

The inspection process involves a human element and is unlikely to be perfect. Decisionerrors will be made from time to time, potentially reflecting sampling and non-sampling sources of error. The inspection game literature (Rothenstein and Zamir, 2002, see, for example) highlights two types of decision-errors in the inspection process, both of which are of interest for biosecurity inspections. We assume the Type One $(\epsilon)$ and Type Two $(\delta)$ Error probabilities are determined exogenously ${ }^{15}$ and known to both the regulator and importer.

A Type One Error involves the regulator raising a "false alarm" by claiming biosecurity risk material is present in a consignment when it is not. ${ }^{16}$ A false alarm could result in a consignment being flagged for further testing to determine whether the "foreign" material is endemic to the domestic market. Such occurences reflect difficulties in identifying the

\footnotetext{
${ }^{13}$ In this setup, we implicitly assume the regulator can observe whether an uninspected consignment contained biosecurity risk material after the fact, akin to holding a costless leakage survey with perfect detection.

${ }^{14}$ In practice, biosecurity risk material leaking into the environment may not always result in a pest or disease becoming established. The leakage cost parameter, $\gamma$, in this model can be interpreted as the "expected" cost of risk material leaking into the environment.

${ }^{15} \mathrm{~A}$ potential extension of this framework could assess the trade-off between inspection intensity, investigated in Fernandez and Sheriff (2013), and the probability of a Type Two Error. This would allow the cost of inspection for the importer $(\omega)$ to be endogenously determined.

${ }^{16}$ The "null hypothesis" of the regulator is that the consignment is free from biosecurity risk material.
} 
status of foreign material and could be affected by caution on the regulator's part to avoid leakage. Type One Errors result in the importer incurring the additional cost $\eta<\kappa$ related to further testing of the good on top of the cost of being inspected $(\omega)$; the regulator incurs an additional social cost of $\tau<\gamma$ from undertaking additional, ultimately unnecessary, testing on "clean" consignments.

Type Two Errors are much more serious and the worst possible outcome from the regulator's perspective. This error results in a consignment containing biosecurity risk material passes inspection without being treated, potentially causing significant damage to the local economy or environment. In this situation, the regulator incurs both the consequences of leakage $(\gamma)$ and the cost of completing an inspection $(\chi)$, while the importer only incurs the cost of being inspected $(\omega)$.

Figure 1 shows the inspection game described above, where the probability of the different moves by "Nature" is affected by the vertically integrated importer's choice of abatement effort, $a$. In the remainder of this section, we describe the subgame-perfect Nash equilibria of the model and illustrate some important features of the model via comparative statics. The solution, in general, is complicated because of corner solutions stemming from:

- the importer's effort having to be non-negative;

- the inspection probability lying between zero and one; and

- the participation constraint on the importer's activities.

The Online Appendix describes the full algebraic solution of the model, together with some illustrative examples.

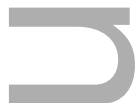

\section{ii Importer's best response}

The first step in solving for the game's subgame-perfect Nash equilibria involves determining the importer's best response correspondence as a function of the inspection 


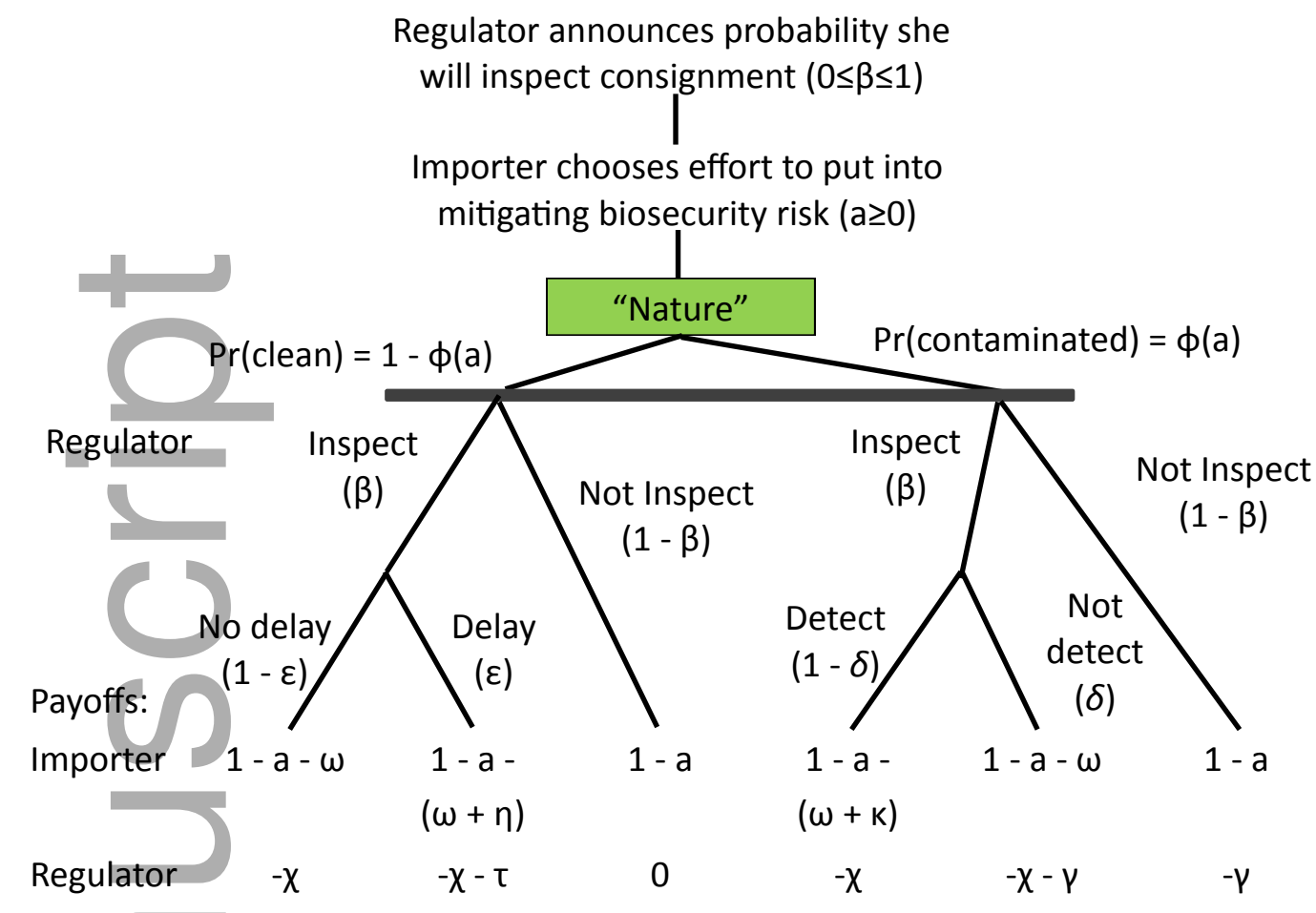

Figure 1: Extensive-form representation of the one-shot biosecurity inspection game allowing for decision-errors.

probability $\beta$ and inspection cost $\omega$ influenced by the regulator. The importer's expected profit function is given by:

$$
U(a)=1-a-\beta[\omega+\epsilon \eta+((1-\delta) \kappa-\epsilon \eta) \phi(a))]
$$

To constrain our attention to economically interesting cases, we assume $(1-\delta) \kappa-\epsilon \eta>0$, so (1) is concave in abatement effort and not monotonically decreasing over $a \geq 0$. Implicitly, the participation constraint on the importer means that $a$ is bounded above by one.

Figure 2 illustrates the geometry of the importer's best-response strategy assuming the regulator does not make decision-errors $(\delta=\epsilon=0)$. The top right-hand panel of this figure shows the optimal strategy $a^{*}(\beta)$ described with a thick black line. Note that if the non-negativity constraint on the importer's level of effort is ignored, (1) is maximised 


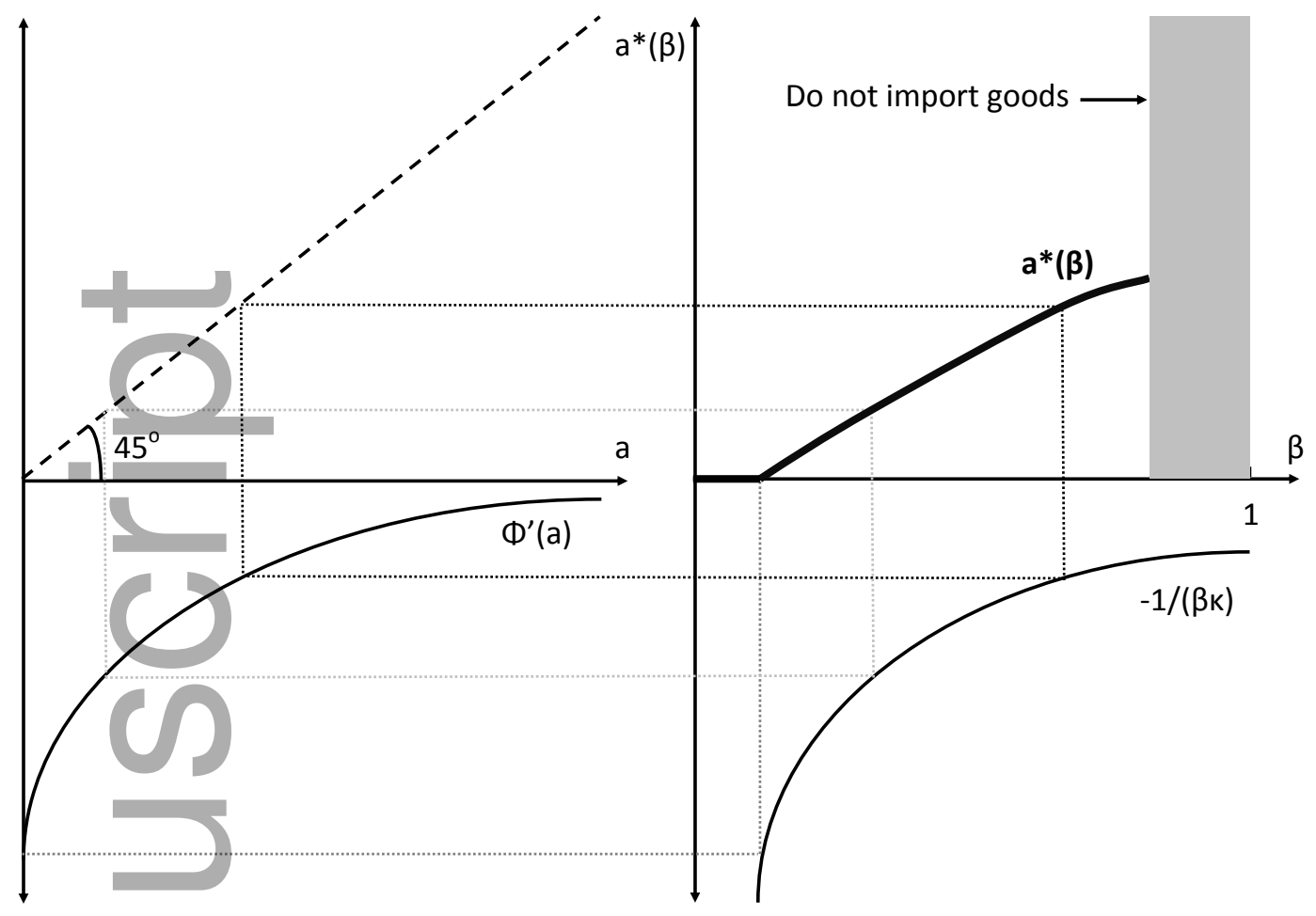

Figure 2: Geometric representation of the importer's best-response strategy in the biosecurity inspection game assuming perfect inspection.

when $a^{*}$ satisfies:

$$
\phi^{\prime}\left(a^{*}\right)=-\frac{1}{\beta \kappa}
$$

The two parts of the lower panel of Figure 2 show the geometry of determinants of this first-order condition for optimaliity, with the lower left- and right-hand panels corresponding to the left- and right-hand sides of (2) respectively. The top left-hand panel simply represents the relationship $f(a)=a$ by a 45 -degree line to translate the solution of the first-order condition into $\beta-a^{*}(\beta)$ space.

Figure 2 shows the importer's effort is increasing with the regulator's announced probability of inspection $\beta$; similarly, optimal effort is also increasing in the treatment cost $\kappa$ for rectifying a consignment containing biosecurity risk material. The marginal condition described in (2) may not be satisfied for some values of $\beta$, as a must be non-negative. For values of $\beta$ satisfying:

$$
\beta \leq-\frac{1}{\kappa \phi^{\prime}(0)},
$$


(1) is monotonically decreasing over $a \geq 0$, so that $a^{*}=0$ represents the constrained optimum. This corner solution is represented by the "flat section" in the plot of $a^{*}(\beta)$ in the top right-hand panel of Figure 2. ${ }^{17}$ Note that our framework can accommodate the situation where it is cheaper for the importer to leave all the biosecurity assurance effort to the regulator at the border. For example, if $\phi(a)$ satisfies:

$$
\phi^{\prime}\left(a^{*}\right)>-\frac{1}{\kappa}, \quad a \in[0,1],
$$

then the importer will maximise expected profit by exerting no effort in cleaning the consignment pre-border. In effect, this leaves "cleaning" to the regulator as part of the inspection process.

Figure 2 also shows the influence of the participation constraint $\left(U\left(a^{*}(\beta)\right) \geq 0\right)$ on the importer's actions. The shaded area in the top right-hand panel indicates the range of inspection probabilities over which the importer would choose not to import the good, as the expected return from importing is negative. To clarify this notion, if zero effort is exerted by the importer, ${ }^{18}$ then the vertically integrated importer will only import the good if the inspection probability satisfies:

$$
\beta \leq \frac{1}{\omega+\kappa \phi(0)} .
$$

The participation constraint may not bind for some types of importers; however, the regulator's conduct can determine costs associated with the inspection process, implying the regulator's choices of inspection fees may influence the nature of goods brought into the country.

When introducing decision-errors, some of the model's qualitative predictions change. In place of (2), the first-order condition for the unconstrained optimisation problem be-

\footnotetext{
${ }^{17}$ If the abatement technology function satisfies $\phi^{\prime}(a) \rightarrow-\infty$ as $a \rightarrow 0^{+}, a^{*}(\beta)$ will be strictly increasing; see the Online Appendix for an example of this form.

${ }^{18}$ The solution in the zero-effort case represents an upper bound on the inspection probability ceiling where strictly positive abatement effort is induced.
} 
comes:

$$
\phi^{\prime}\left(a^{*}\right)=-\frac{1}{\beta((1-\delta) \kappa-\epsilon \eta)},
$$

which is akin to changing the requirement line in the bottom right-hand panel of Figure 2. Figures 3 and 4 illustrate the separate effects of the Type One and Type Two errors, respectively, on the importer's best-response strategy.

For the Type One Error, Figure 3 shows the discontinuity introduced in the right-hand side of (3), with a vertical asymptote introduced at $\beta=\frac{\epsilon \eta}{\kappa} \cdot{ }^{19}$ By pushing out the requirement line in the bottom right-hand panel, the importer is better off exerting less effort in cleaning the consignment relative to the situation without decision-errors. This results in the response $a^{*}=0$ being optimal over a larger interval of inspection probabilities. Since the importer incurs additional costs from the regulator raising "false alarms", the participation constraint binds at lower values of $\beta$, implying a larger range of inspection probabilities over which the importer would choose not to import.

Figure 4 demonstrates introducing a Type Two Error has a similar impact on the importer's best-response as a lower treatment cost $(\kappa)$. Similar to the Type One Error, introducing the possibility the regulator fails to detect a consignment containing biosecurity risk material results in the importer reducing the abatement effort they exert. Unlike the case with a Type One Error, a Type Two Error shrinks the window over which the participation constraint binds, because the costs the importer expects to incur are lower if there is a chance a contaminated consignment avoids treatment.

\section{iii Regulator's inspection probability choice}

In considering the solution to the regulator's optimisation problem, we assume the importer's participation constraint is not binding for $\beta \in[0,1]$. The regulator chooses $\beta$ to

\footnotetext{
${ }^{19}$ The discontinuity induces a segment over which the derivative of $U(a)$ is decreasing, which may result in $a^{*}=0$ being optimal over a greater range of inspection probabilities than suggested in Figure 3. See the Online Appendix for details.
} 


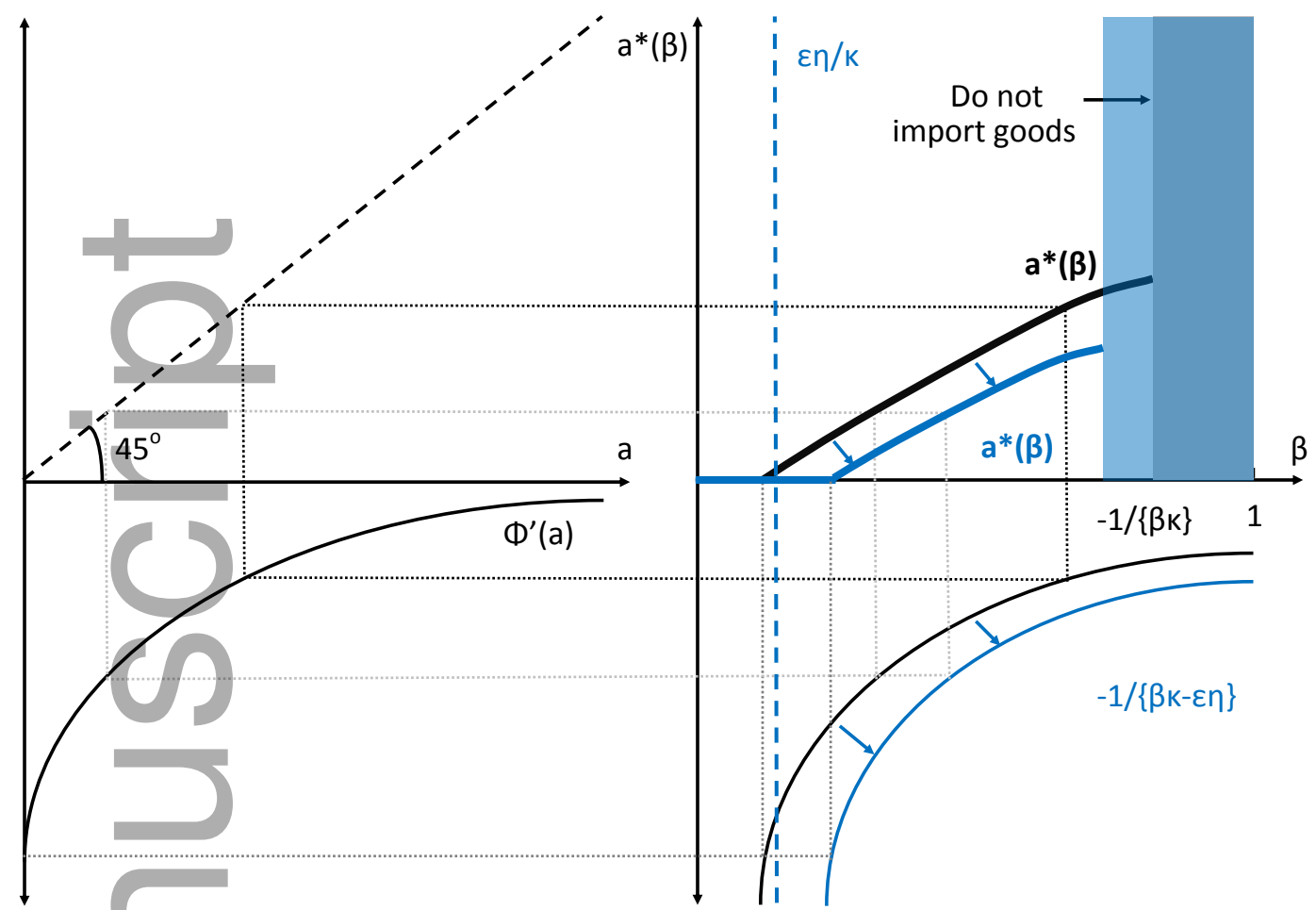

Figure 3: Geometric representation of the importer's best-response strategy under a Type One Error in inspection relative to a perfect inspection process.

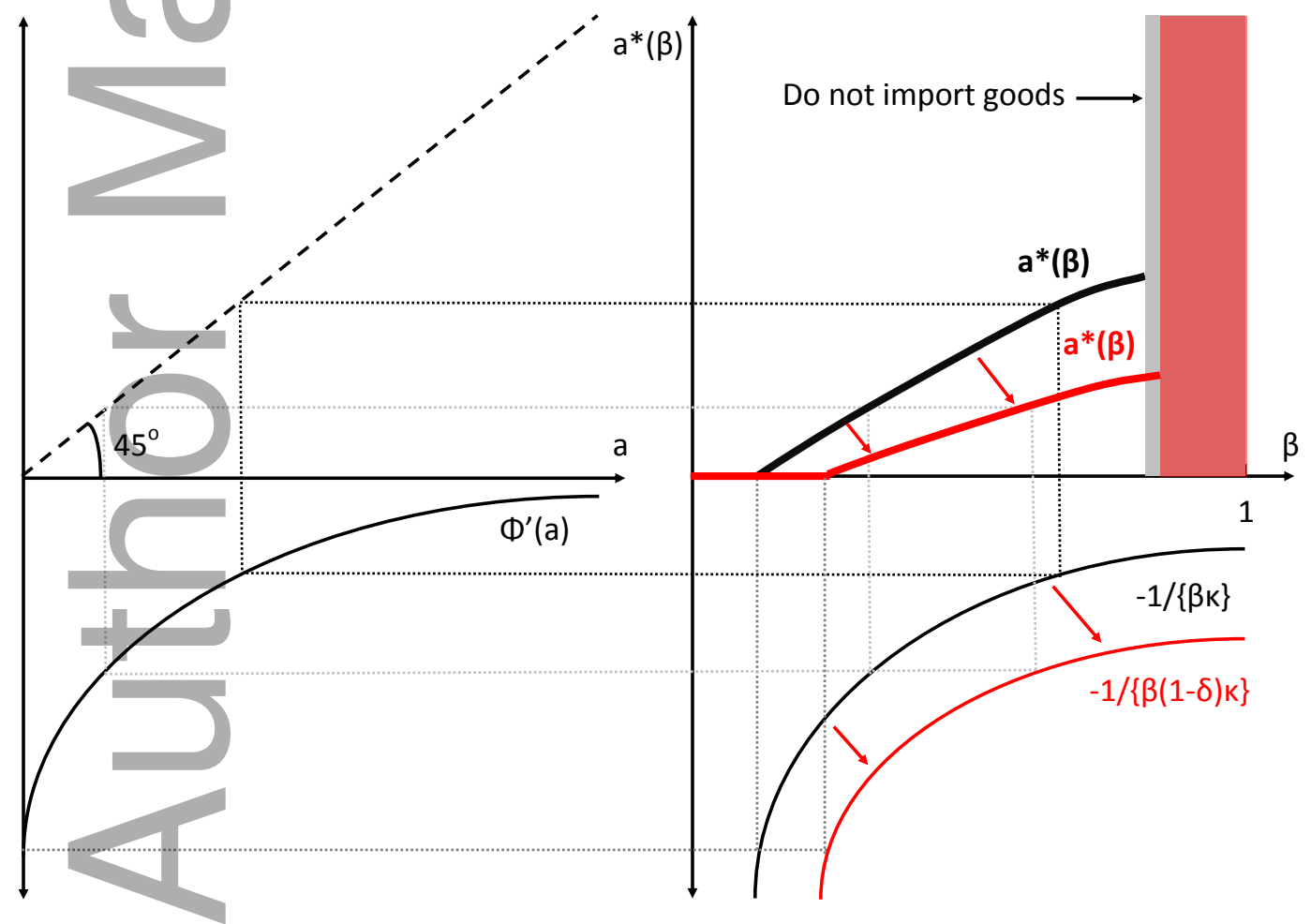

Figure 4: Geometric representation of the importer's best-response strategy under a Type Two Error in inspection relative to a perfect inspection process. 
maximise:

$$
V\left(a^{*}(\beta), \beta\right)=\beta\left[\phi\left(a^{*}(\beta)\right)((1-\delta) \gamma+\epsilon \tau)-(\chi+\epsilon \tau)\right]-\phi\left(a^{*}(\beta)\right) \gamma
$$

where the regulator, as the first-mover in the game, takes into account the importer's best-response correspondence, $a^{*}(\beta)$. In general, solving this optimisation problem is challenging because $a^{*}(\beta)$ is only piecewise differentiable. This means considering several cases that depend on the functional form of the abatement technology function and the key cost parameters for the regulator relating to leakage $(\gamma)$ and undertaking an inspection $(\chi)$.

Figure 5 illustrates two principal cases of interest - one where the importer exerts zero effort regardless of the stated inspection probability (panel a) and the other where the importer exerts strictly positive effort in equilibrium (panel b). If it is optimal for the importer to apply zero abatement effort regardless of the inspection probability chosen by the regulator, panel (a) shows the regulator's optimal inspection probability announcement is an "all or nothing" decision. The threshold between not inspecting or inspecting with certainty in terms of the cost of leakage is given by:

$$
\gamma^{*}=\frac{\chi+(1-\phi(0)) \epsilon \tau}{\phi(0)(1-\delta)}
$$

the regulator is indifferent between inspecting and not inspecting if $\gamma=\gamma^{*}$. Under a perfect decision-making environment for inspections, the threshold (5) has the convenient interpretation of the regulator balancing the expected cost of leakage (i.e. $\phi(0) \gamma)$ with the cost of conducting an inspection $(\chi)$. In a sense, this provides a "new" interpretation of an appropriate level of protection for the regulator.

In equilibrium, the regulator would choose not to inspect a consignment with a higher threshold consequence of leakage when decision-errors are introduced compared to a per- 
(a)

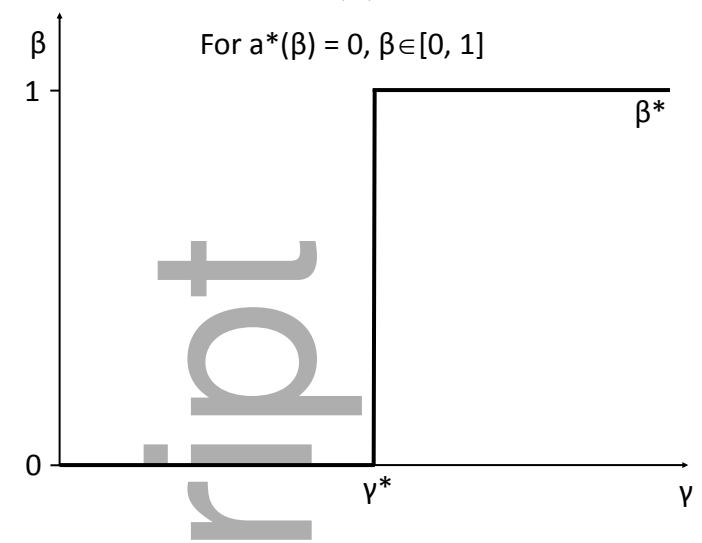

(b)

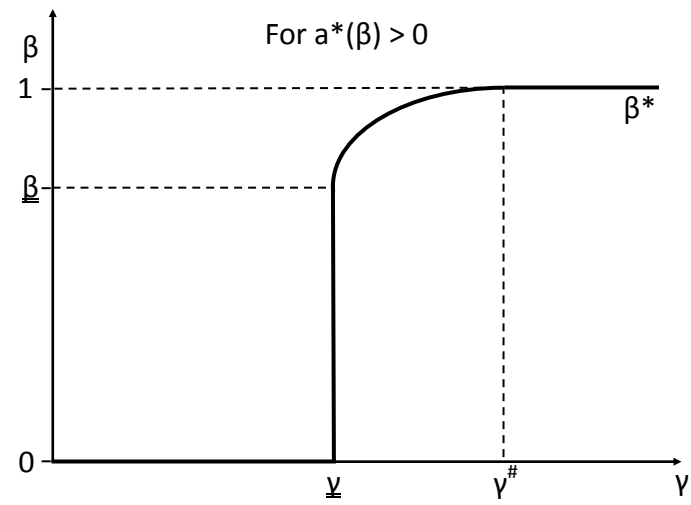

Figure 5: Geometric representation of the regulator's optimal choice of inspection probability given by the game's subgame-perfect Nash equilibrium under: (a) no effort exerted by the importer for any announced inspection probability; and (b) strictly positive effort exerted by the importer.

fect inspection process $(\delta=\epsilon=0)$. This is because:

$$
\gamma^{*}=\frac{\chi+(1-\phi(0)) \epsilon \tau}{\phi(0)(1-\delta)} \geq \frac{\chi}{\phi(0)}=\underset{\sim}{\gamma}
$$

with the inequality strict if $\delta>0$ and/or $\epsilon>0$ and $\tau>0$. This apparently counterintuitive result reflects the lower value of conducting inspections for detecting biosecurity risk material $(1-\delta)$ and the expected costs borne by the regulator in delaying "clean" consignments $(\epsilon \tau)$.

If there are values of $\beta$ for which the importer exerts positive abatement effort, the inspection game's subgame-perfect Nash equilibrium depends on the relative magnitudes of specific model parameters and the precise functional form of the abatement technology function. Panel (b) of Figure 5 illustrates the general pattern of the regulator's inspection probability choice when the importer's best response is to exert strictly positive abatement effort. For some parameter values for this model, it is optimal for the regulator to select an "intermediate" inspection probability strictly between zero and one to equalise their trade-offs. The effect of introducing decision-errors in inspection on the subgameperfect Nash equilibrium is also ambiguous in general and depends on the properties of the abatement technology function. The Online Appendix contains some worked exam- 
ples and discusses technical conditions associated with solving the subgame-perfect Nash equilibrium for the game.

\section{Adaptive inspection rules from the continuous sampling plan family}

The previous section considered the biosecurity inspection process as a one-off interaction. In practice, these interactions are repeated, often at fairly regular intervals, as importers bring in successive consignments from overseas. Adaptive inspection rules, which enable importers with good compliance records to be subject to a lower frequency of inspection, have been introduced for select plant-based products in Australia. These rules possess default incentive structures that may create positive or perverse incentives for importer compliance. This section describes three adaptive inspection rules from the CSP family, one of which - the CSP-3 algorithm - is currently used by the DAWR.

The most basic of the CSP family rules is the CSP-1 algorithm, which was introduced in Dodge (1943) and is illustrated in Figure 6. When a new importer starts on this algorithm, they are usually subject to mandatory inspections (in "census mode") until they build up a good compliance record. Two key parameters for the regulator to choose in this rule are:

- the clearance number $C N$ - the number of consecutive consignments that must pass inspection for the importer to be eligible for a reduced inspection frequency; and

- the monitoring fraction, $M F$ - the reduced inspection frequency; that is, the probability a given consignment is inspected in "monitoring mode".

If an importer's consignment fails inspection when the importer is in "monitoring mode", their subsequent consignments are subject to mandatory inspection in "census" mode. 


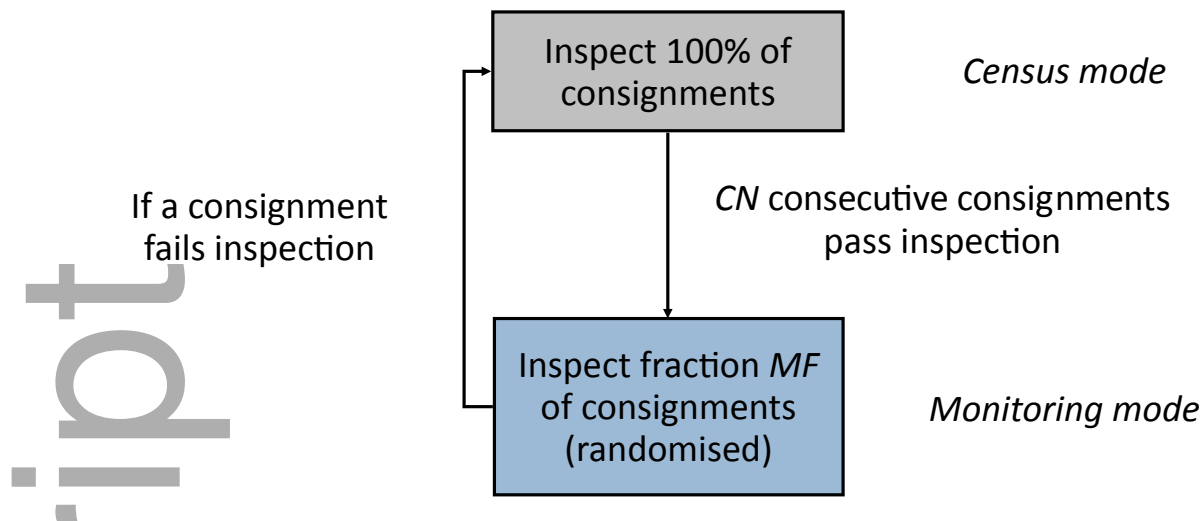

Figure 6: CSP-1 algorithm representation.

The importer only receives the reduced inspection frequency again after another $C N$ consecutive consignments pass inspection.

The CSP-2 and CSP-3 rules documented in Dodge and Torrey (1951) have less severe consequences for occasional non-compliance when an importer is on the reduced inspection frequency $M F$ relative to the CSP-1 rule. In the CSP-2 algorithm (Figure 7), if an importer's consignment fails inspection in monitoring mode, they continue to be inspected at the reduced rate $M F$ while the regulator keeps track of the number of inspections passed since the last recorded failure. This part of the algorithm is usually referred to as "failure detection mode". Provided the importer passes inspection $C N$ times since their last failure, they remain eligible to be inspected at the reduced rate of inspection; otherwise, on recording another failure within $C N$ consignments of the previous one, the importer's consignments revert to mandatory inspection until they pass inspection $C N$ times in a row. Intuitively, this provides less of a "cost" to the importer if recording a failure in one inspection does not increase the probability that future consignments will be more likely to fail.

The CSP-3 algorithm, shown in Figure $8,{ }^{20}$ adds another layer of complexity to the CSP-2 algorithm. This is designed to provide extra protection to the regulator against a sudden systematic problem that would significantly raise the likelihood of a consignment failing

${ }^{20}$ The version of the rule used in this paper follows the practical simplification suggested by Robinson et al. (2012). 


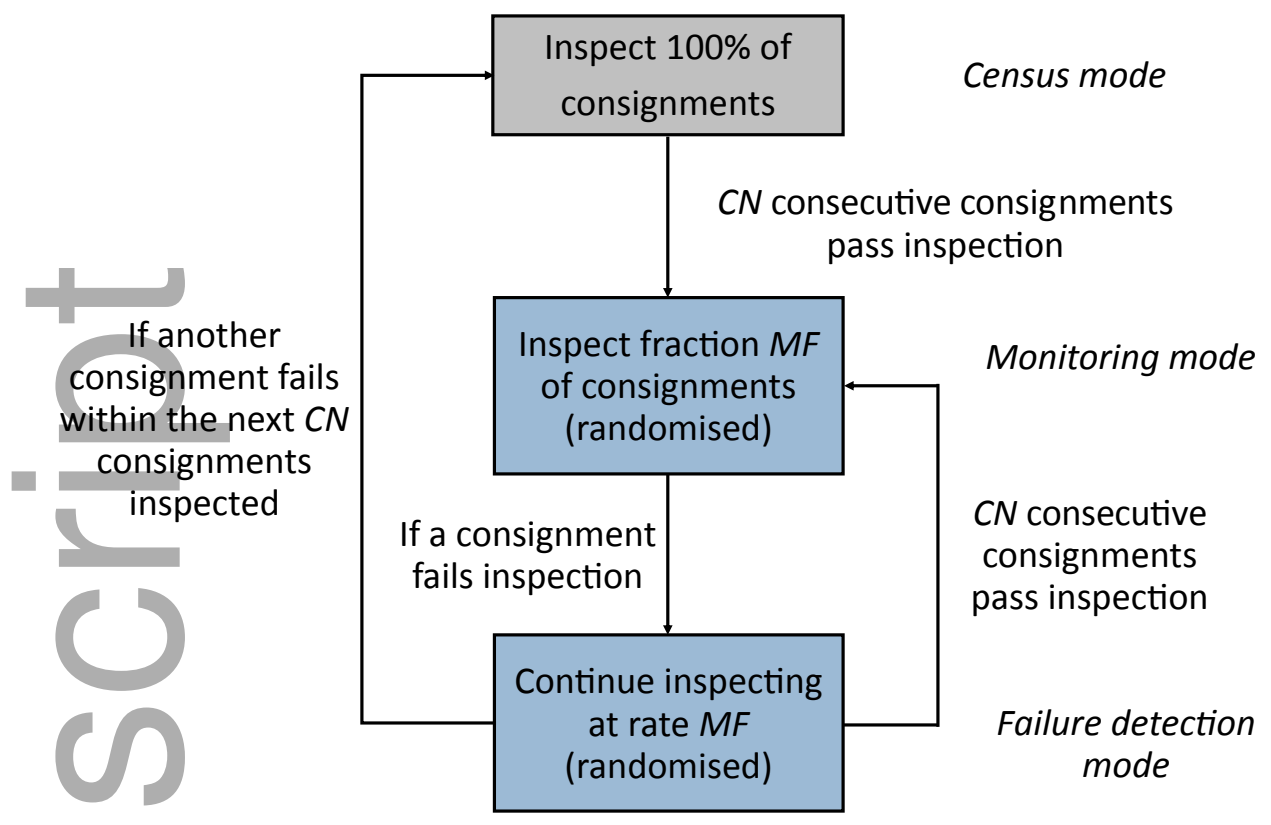

Figure 7: CSP-2 algorithm representation.

inspection. It does this by making the next four consignments following a failure subject to mandatory inspection in what is referred to as "tight census mode". The other features of the CSP-2 algorithm, such as ignoring past failures if they occurred more than $C N$ inspections ago, are retained by the CSP-3 algorithm.

\section{Dynamic importing game with fixed and variable technology choices}

\section{i Game specification}

The one-shot biosecurity inspection game described in Section II provided insights into some of the influences likely to affect interactions between a vertically integrated importer and biosecurity regulator. From the importer's perspective, a higher treatment cost encourages them to increase the effort they apply pre-border to cleaning consignments, while decision-errors from the regulator discourage the application of abatement 

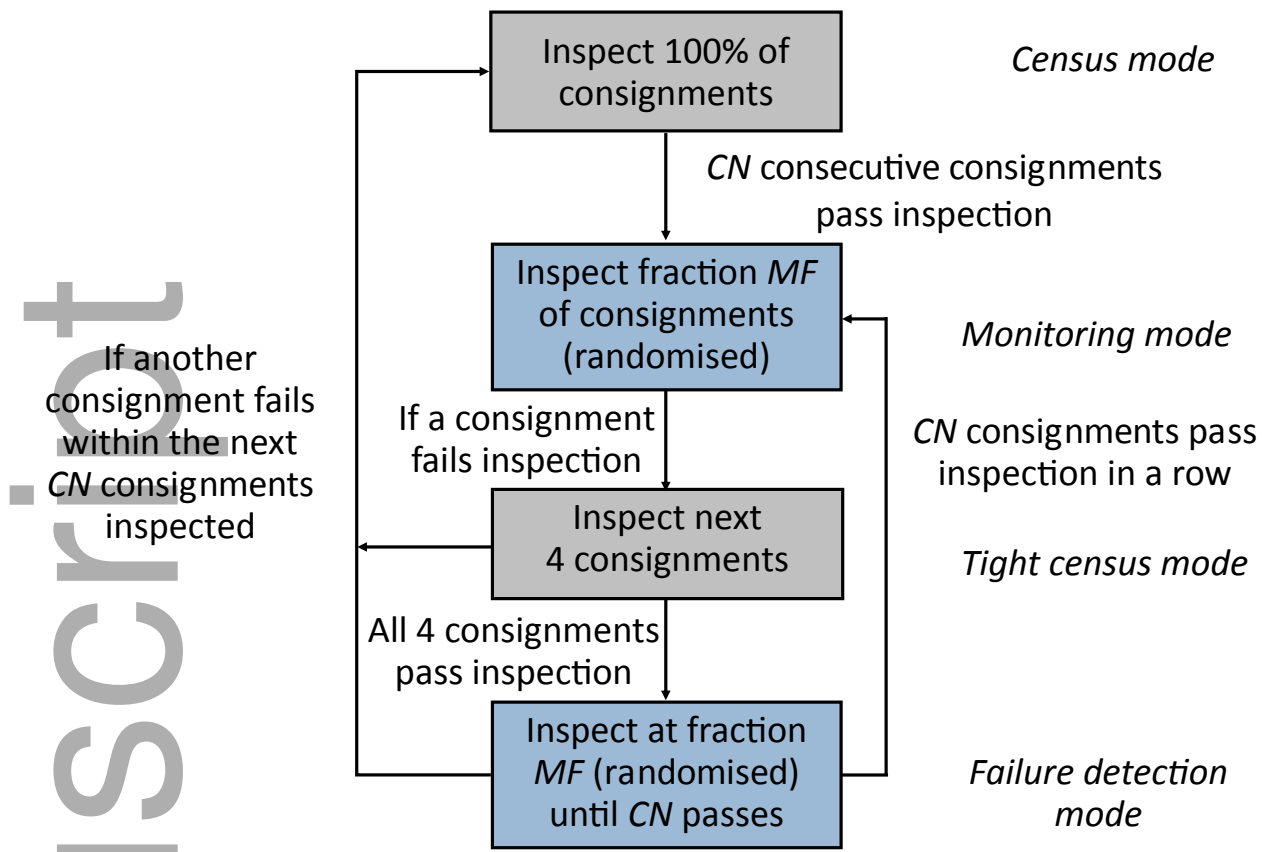

Figure 8: CSP-3 algorithm representation.

effort. The cost of inspection did not directly influence the importer's choice of abatement effort in the one-shot case, though it affected whether or not they brought in the good to the domestic market. For the regulator, the subgame-perfect Nash equilibrium inspection probability was determined predominantly by the relative costs of completing an inspection and the consequences of biosecurity risk material leakage; higher relative costs of leakage beyond a given threshold resulted in the regulator choosing to inspect with probability one.

When the inspection interaction is repeated over time, the importer's optimal behaviour is likely to differ from the one-shot game's best-response effort levels corresponding to either $\beta=1$ or $\beta=M F$. The adaptive rules considered in this paper, where an importer's past choices can affect the payoffs currently available to them, provide additional dependencies over time that the importer needs to account for when they determine their optimal strategy. Furthermore, the importer's production processes might mean that not all forms of "effort" can be freely manipulated in each period because of technological choices that have longer-run considerations. 
The dynamic model in this section accounts for these features by allowing a vertically integrated importer to make an upfront one-off choice of an irreversible, fixed investment that governs a component of their abatement technology function. From a contract theory perspective, this decision governs the importer's "type" in the dynamic inspection interaction. Together with the regulator's use of adaptive inspection rules from the CSP family, the use of fixed technologies results in the importer's optimisation problem being a Markov decision process; see the Online Appendix for more details of this specification. The importer's technology choice is subject to increasing marginal cost, with the technology choices associated with lower "baseline" biosecurity risk material approach rates becoming increasingly costly for the importer to implement.

From the regulator's perspective, adaptive inspection algorithms may help tailor the allocation of inspection effort for a particular type of good. Importers better able to comply with biosecurity requirements because of access to superior processes, or inspection cost structures that provide private incentives for compliance, can be inspected less frequently on average than other importers. Such rules can also partly overcome the limited information available to the regulator about a firm's type based on their compliance history. However, care needs to be taken in choosing rule parameters - the clearance number $(C N)$ and monitoring fraction $(M F)$ for rules from the CSP family - to sufficiently separate importers whose characteristics encourage them to be highly compliant from those more likely to import consignments containing biosecurity risk material. Given the biosecurity regulator does not know the importer's abatement technology function in practice, they also need to consider adverse selection-type problems that could arise if inspection rules are poorly calibrated.

A key concern for the regulator with the CSP family of rules is that, once an importer goes into monitoring mode, it may be advantageous for them to reduce the variable effort they apply to cleaning consignments relative to when they are in census mode. This pattern of behaviour is suggested by period-by-period optimisation of the model considered in 
Section II and could result in an appreciably higher approach rate in monitoring mode than in census mode. Whether it is optimal for the importer to display this "cheating" behaviour in monitoring mode will be influenced by:

- the rule's inherent incentives, governed in part by the parameters selected by the regulator;

- characteristics associated with the importer, such as the costs faced in being inspected and of adopting different fixed technologies; and

- characteristics of the inspection process, such as the regulator's decision-error probabilities.

Our dynamic version of the inspection game between the regulator and importer involves several stages.

1. The biosecurity regulator commits to an inspection rule, which consists of a choice of the CSP-1, CSP-2 or CSP-3 algorithm and the parameters $C N$ and $M F$, covering $C$ consignments.

2. The vertically integrated importer chooses an irreversible, fixed investment that governs a fixed parameter $(\theta \in(0,1])$ in its abatement technology function $\phi\left(\theta, a_{t}\right)$, where $\phi(\cdot)$ is increasing in $\theta$. In making the choice of the fixed technology parameter, the importer incurs a cost $c(\theta)$, where $\lim _{\theta \rightarrow 0^{+}} c(\theta)=\infty, \lim _{\theta \rightarrow 0^{+}} c^{\prime}(\theta)=-\infty$ and $c^{\prime \prime}(\theta)>0$.

3. For each consignment $t=1, \ldots, C$, the importer can choose (variable) abatement effort $a_{t}$ and faces a discounted per-period payoff of $U\left(a_{t}, \theta\right)$, with a rate of time preference $\rho \in(0,1)$.

While dynamic programming techniques could be used to solve the importer's optimisation problem for a given rule structure and parameters, this particular model structure does not appear to admit an analytical solution. Furthermore, conventional numerical algorithms that use the dynamic programming formulation, such as value function itera- 


\begin{tabular}{|c|c|c|c|}
\hline $\begin{array}{l}\text { Model Parameter/Function } \\
\text { Description }\end{array}$ & $\begin{array}{l}\text { Representa- } \\
\text { tion }\end{array}$ & $\begin{array}{c}\text { Base Case } \\
\text { Value/Form }\end{array}$ & $\begin{array}{c}\text { Scenario } \\
\text { Value/Form }\end{array}$ \\
\hline \multicolumn{4}{|l|}{ Dynamic model parameters } \\
\hline Total consignments & $\mathrm{C}$ & 2000 & \\
\hline Discount rate & $\rho$ & 0.9996 & \\
\hline \multicolumn{4}{|l|}{ Importer cost parameters } \\
\hline Inspection cost & $\omega$ & 0.3 & 0.1 \\
\hline Treatment cost & $\kappa$ & 0.8 & 1.5 \\
\hline Delay cost (from false alarm) & $\eta$ & 0 & \\
\hline \multicolumn{4}{|l|}{ Abatement technology } \\
\hline function & $\phi\left(a_{t}\right)$ & $\theta \exp \left(-\lambda a_{t}\right)$ & \\
\hline Fixed technology parameter & $\theta \in(0,1]$ & & \\
\hline Variable effort level & $a_{t} \geq 0$ & & \\
\hline Function exponent parameter & $\lambda$ & 25 & 5 \\
\hline Fixed technology cost function & $c(\theta)$ & $\frac{4}{\theta}$ & $\frac{1}{\theta}$ \\
\hline \multicolumn{4}{|l|}{ Regulator cost parameters } \\
\hline \multicolumn{4}{|l|}{ Cost of completing } \\
\hline an inspection & $\chi$ & 0.05 & 0.2 \\
\hline Cost of leakage & $\gamma$ & 1.2 & 3 \\
\hline Social cost of false alarm & $\tau$ & 0 & \\
\hline \multicolumn{4}{|l|}{ Decision-error probabilities } \\
\hline \multicolumn{4}{|l|}{ Type One Error } \\
\hline \multicolumn{4}{|l|}{ Type Two Error } \\
\hline (leakage after inspection) & $\delta$ & 0.05 & 0.20 \\
\hline
\end{tabular}

Table 1: Parameters and functional forms for the dynamic biosecurity inspection interaction.

tion or policy iteration, are challenging to implement for this type of problem because of the large number of states underpinning the Markov decision process and the inequality constraints on the feasible solution space. We therefore revert to stochastic optimisation routines to solve the problem numerically and use a pattern search algorithm to solve for the importer's dynamic problem under different values of $C N, M F$ and the three CSP rules. Through sensitivity analysis, we discuss the impact of the parameters on the appropriateness of different inspection rules available to the biosecurity regulator. The functional forms and parameters for the baseline problem are specified in the third column of Table 1; the fourth column provides the parameters/forms varied one at a time as part of the sensitivity analysis. 
Throughout the simulations in this paper, we use the same number of imported consignments $(C=2000)$ and discount rate $(\rho=0.9996)$. These were calibrated to mimic an importer who brings in 100 consignments a year at regular intervals over 20 years in an economy where the discount rate is around 4 per cent a year.

As the biosecurity regulator is a leader, we solve the importer's optimisation problem for the values of technology parameter $\theta$ and the effort levels $a_{t}$ that maximise their expected payoff for a given CSP rule form and parameters. ${ }^{21}$ To reduce the problem's dimensionality, we also assume the effort values $a_{t}$ are constant in each phase of the CSP algorithm. This means solving for two values of $a_{t}$ with the CSP-1 algorithm (under census and monitoring modes), three for the CSP-2 algorithm (adding failure detection mode) and four for the CSP-3 algorithm (distinguishing tight census from less-intensive component of the failure detection mode).

Given the various parameter choices made by the importer, the regulator then determines the rule from the suite of parameter values that minimises their expected costs of intervening in the market, reflecting the costs of inspection and leakage they internalise. The grid-like approach makes sense for considering the practical implementation of these rules, since the clearance number is integer-valued and the monitoring fraction is likely to be chosen to have a straightforward decimal or fraction representation so it can be easily communicated to stakeholders.

\section{ii Base case}

From the importer's perspective, a reduced rate of inspection is always desirable for improving the profitability of their importing activity. Figure 9 suggests the importer's payoff is higher under a lower clearance number and/or a lower monitoring fraction,

\footnotetext{
${ }^{21}$ The "optimal" solution is based on 200 replications to evaluate the importer's objective function. The numerical procedure appears to work well, but occasionally creates what appear to be discontinuities in some of the relationships. Throughout this section, we focus attention on findings that appear robust to perceived discontinuities.
} 


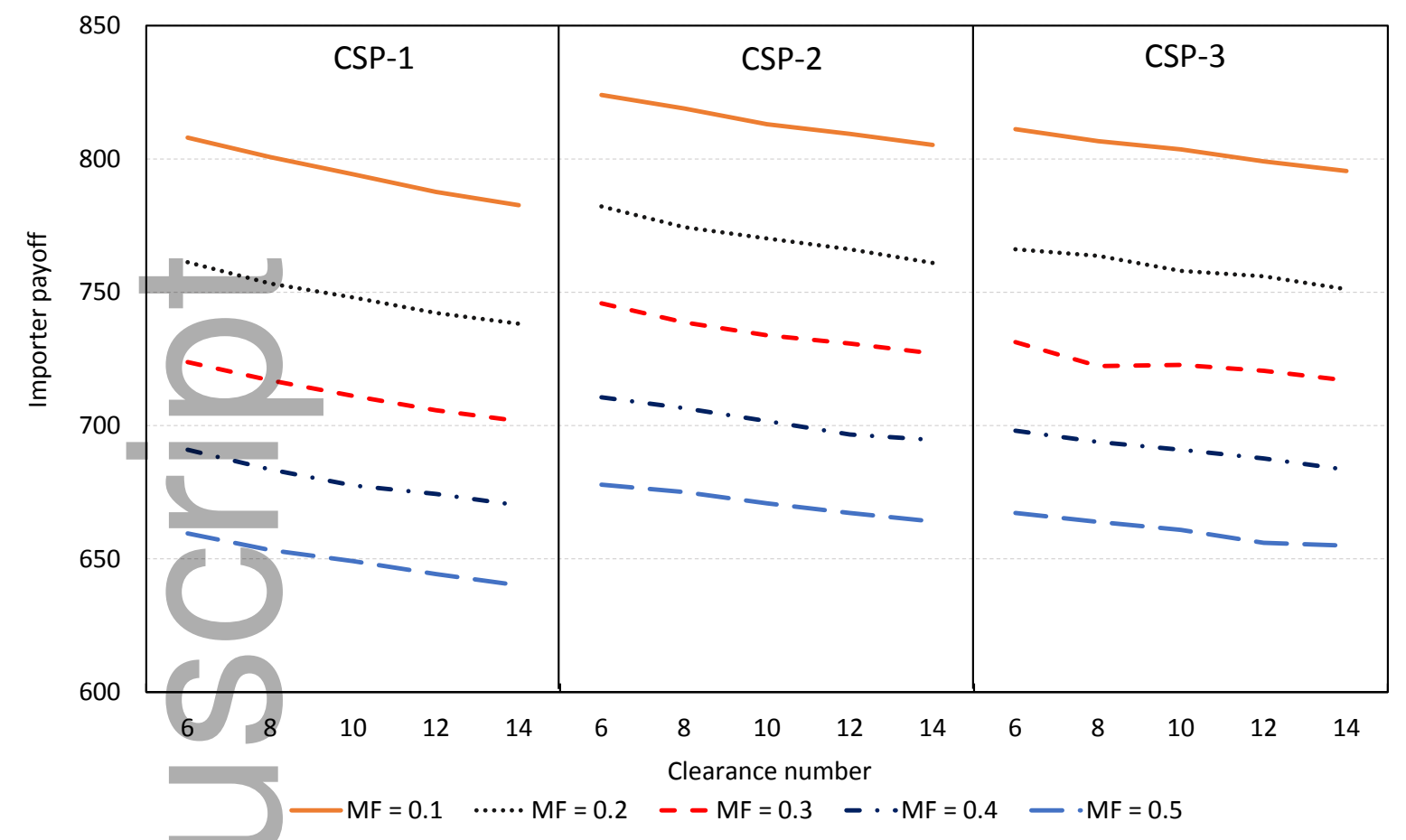

Figure 9: Importer payoffs under base case simulations for CSP-1, CSP-2 and CSP-3 rules.

with payoffs generally highest under the CSP-2 algorithm (middle panel) and lowest for the CSP-1 algorithm (left-hand panel) for given values of the clearance number and monitoring fraction. This aligns with the theoretical properties of the Markov chains for the respective rules discussed in the Online Appendix.

As the regulator specifies the inspection rule, their preferences will determine the rule the importer will face. In doing so, the regulator takes into account the importer's strategic response to the rule chosen. Figure 10 shows the regulator's payoffs under alternative rules in the CSP family, together with the payoff under a mandatory inspection regime. In the base case, the regulator has rules available from the CSP family that reduce the level of intervention and result in a higher payoff for the regulator relative to imposing a mandatory inspection regime. In general, the rules delivering a superior payoff to the mandatory inspection regime have higher monitoring fractions and clearance numbers and tend to follow either the CSP-1 or CSP-3 form. Of the rules assessed in our grid search, the CSP-1 rule with clearance number 14 and monitoring fraction 0.5 provides 


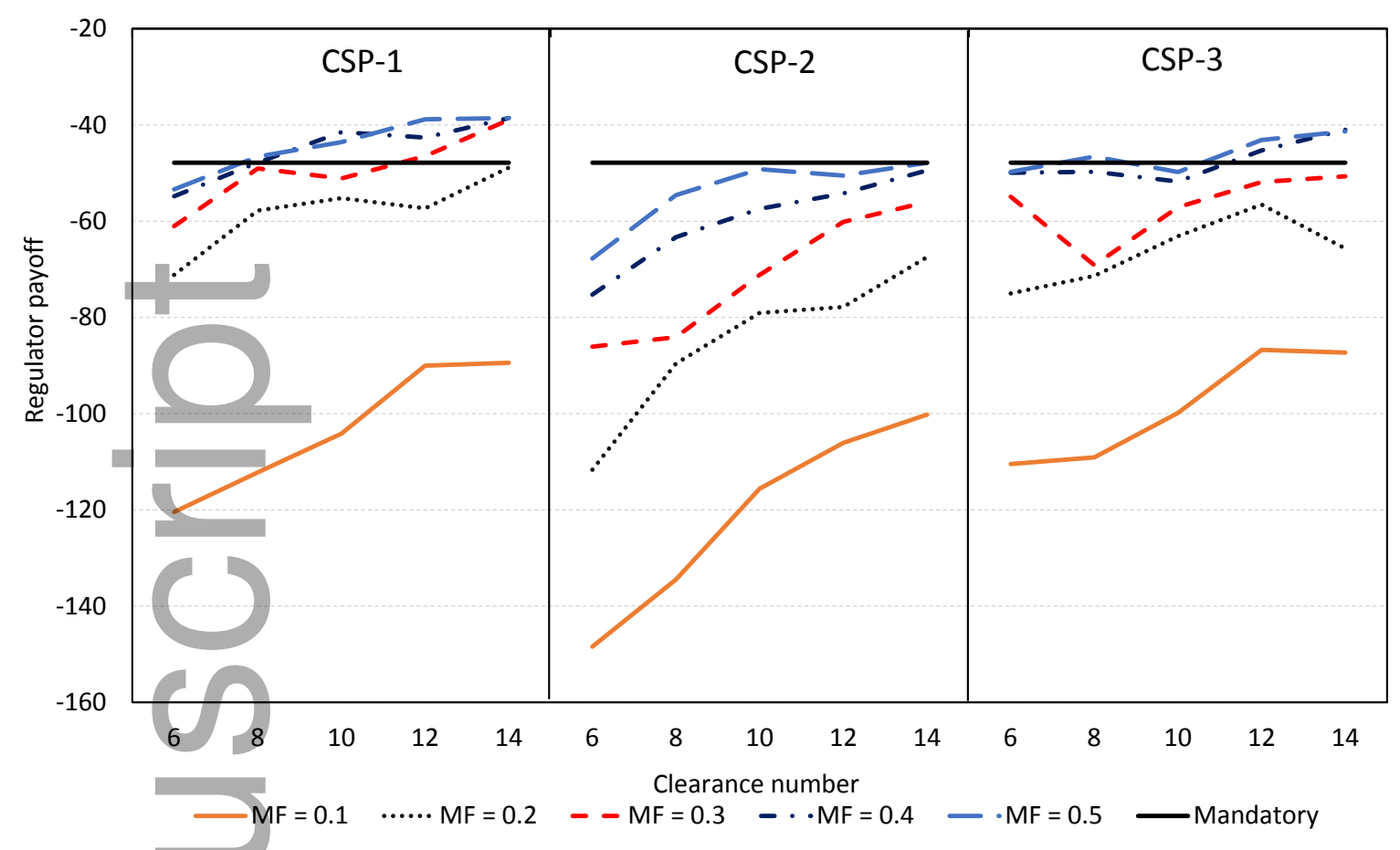

Figure 10: Regulator payoffs under base case simulations for CSP-1, CSP-2 and CSP-3 rules.

the highest payoff to the regulator.

Under the base case parameterisation, variable effort is highly effective in reducing the probability that a consignment contains biosecurity risk material. As a consequence, the importer's best response to being offered a rule with a low monitoring fraction is to use more variable effort under the census mode and substantially reduce their effort in monitoring mode - often to zero. This results in a much higher approach rate in monitoring mode - a type of behaviour the regulator would not want to encourage. As a result, the regulator incurs significant costs associated with biosecurity risk material leaking into the environment in monitoring mode.

A higher monitoring fraction in the CSP rule induces the importer to reduce the biosecurity risk material approach rate relative to the mandatory inspection case under both monitoring and census modes, as illustrated in Figure 11 for the CSP-1 algorithm. A higher clearance number raises the cost of being caught by the regulator and tends to encourage the importer to reduce the biosecurity risk material approach rate in monitoring 


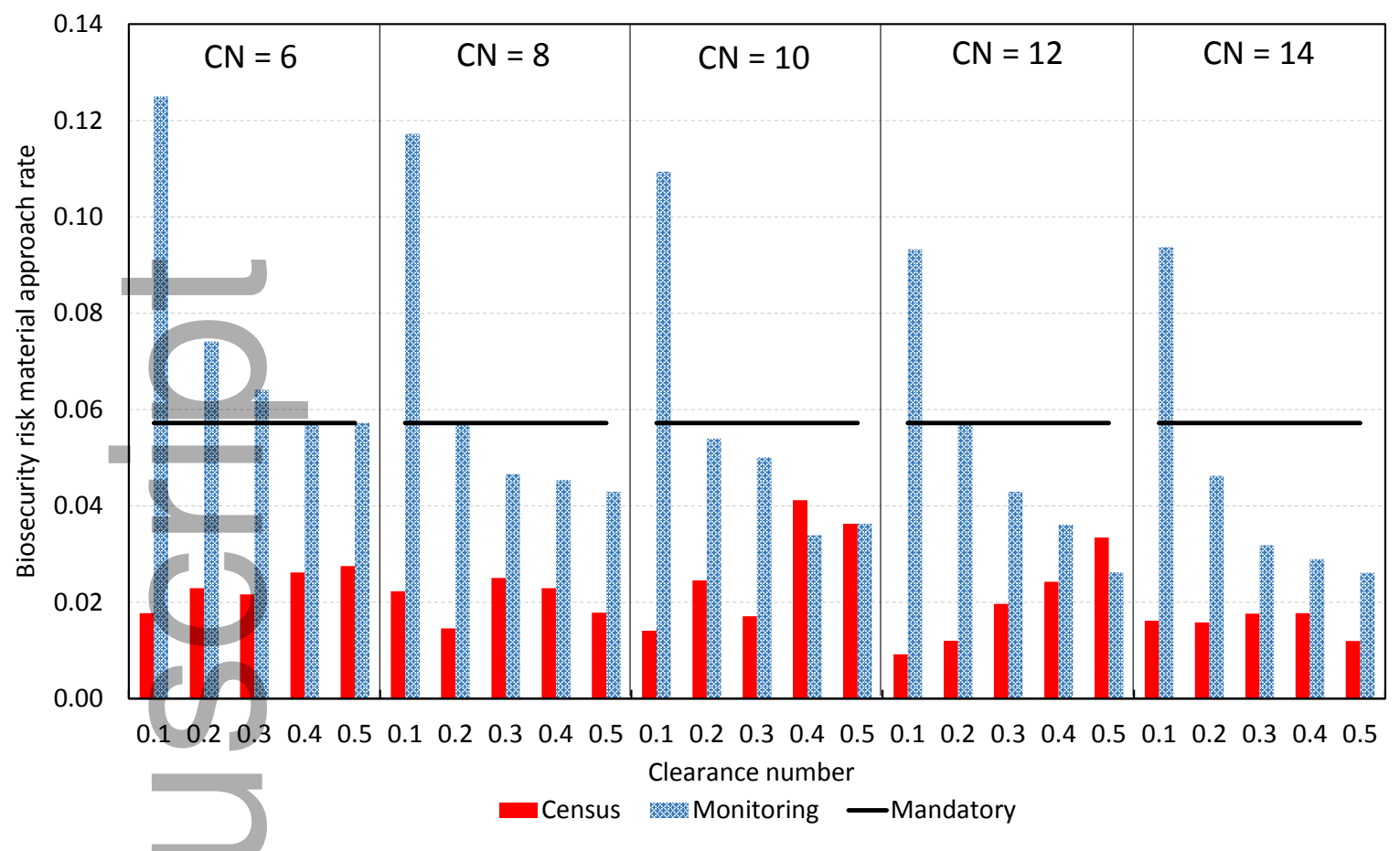

Figure 11: Base case probability of biosecurity risk material contamination based on the importer's "optimal" response under alternative CSP-1 algorithm parameterisations.

mode; however, the incentive for the importer to "cheat" in monitoring mode undermines the use of a high clearance number alone as a suitable approach for the regulator. Thus, a high clearance number and monitoring fraction are required in combination to meet the regulator's requirements.

\section{iii Scenarios: abatement technology-related parameters}

We consider the influence of two dimensions related to technology choices and costs on the appropriateness of different inspection rules for the regulator by considering changes to:

1. the relative effectiveness of variable effort $\left(a_{t}\right)$ and the fixed technology $(\theta)$ in reducing the approach rate of biosecurity risk material. This is done by assessing what occurs for a lower value of $\lambda$, the exponent of $a_{t}$ in the abatement technology function $\phi\left(a_{t}\right)=\theta \exp \left(-\lambda a_{t}\right)$ defined in Table 1 ; and 
2. the relative cost of the fixed technology, $\theta$, to the importer, through proportionally scaling down the cost function $c(\theta)$ from the base case by a factor of four.

The desirability of "cheating" for the importer relies on variable effort being highly effective in reducing the biosecurity risk material approach rate in the census mode of the CSP algorithms. When the marginal effect of effort on the approach rate is lower, the importer relies more on the fixed technology to lower the likelihood of biosecurity risk material contamination. Figure 12 illustrates the importer choosing higher-cost investments in fixed technology (corresponding to lower values of the fixed technology parameter, $\theta$ ) in the scenario (cross-hatched columns in the left-hand panel) while also choosing lower levels of the now relatively less effective variable effort (right-hand panel) compared to the base case. Unlike the base case, these choices mean "cheating" is less of a concern for the regulator in this scenario. While the importer's fixed technology and variable effort choices result in a lower biosecurity risk material approach rate than the mandatory inspection regime for some CSP rule parameters, Figure 13 confirms that a mandatory inspection regime delivers a higher expected payoff for the regulator relative to the CSP rules considered in the grid search.

When the fixed technology is less costly to purchase, the importer has a reduced tendency to "cheat" when a reduced inspection rate is applied in monitoring mode relative to the base case (Figure 14). This reflects that variable effort is both relatively more costly and has a lower marginal impact on reducing the approach rate of biosecurity risk material in this particular specification of the abatement technology function. ${ }^{22}$

Under this scenario, Figure 15 shows that the regulator can improve upon a mandatory inspection regime by choosing from CSP family rules over a much wider range of values for $C N$ and $M F$, including a monitoring fraction as low as 0.2 . Furthermore, the consequences of a regulator making a "mistake" and choosing a low monitoring fraction

\footnotetext{
${ }^{22}$ The latter holds because the partial derivative of $\phi\left(\theta, a_{t}\right)$, with respect to effort $\left(a_{t}\right)$ is proportional to the fixed technology parameter $(\theta)$. With reductions in $\theta$ being less costly, a lower value of $\theta$ reduces the marginal effect of raising effort.
} 


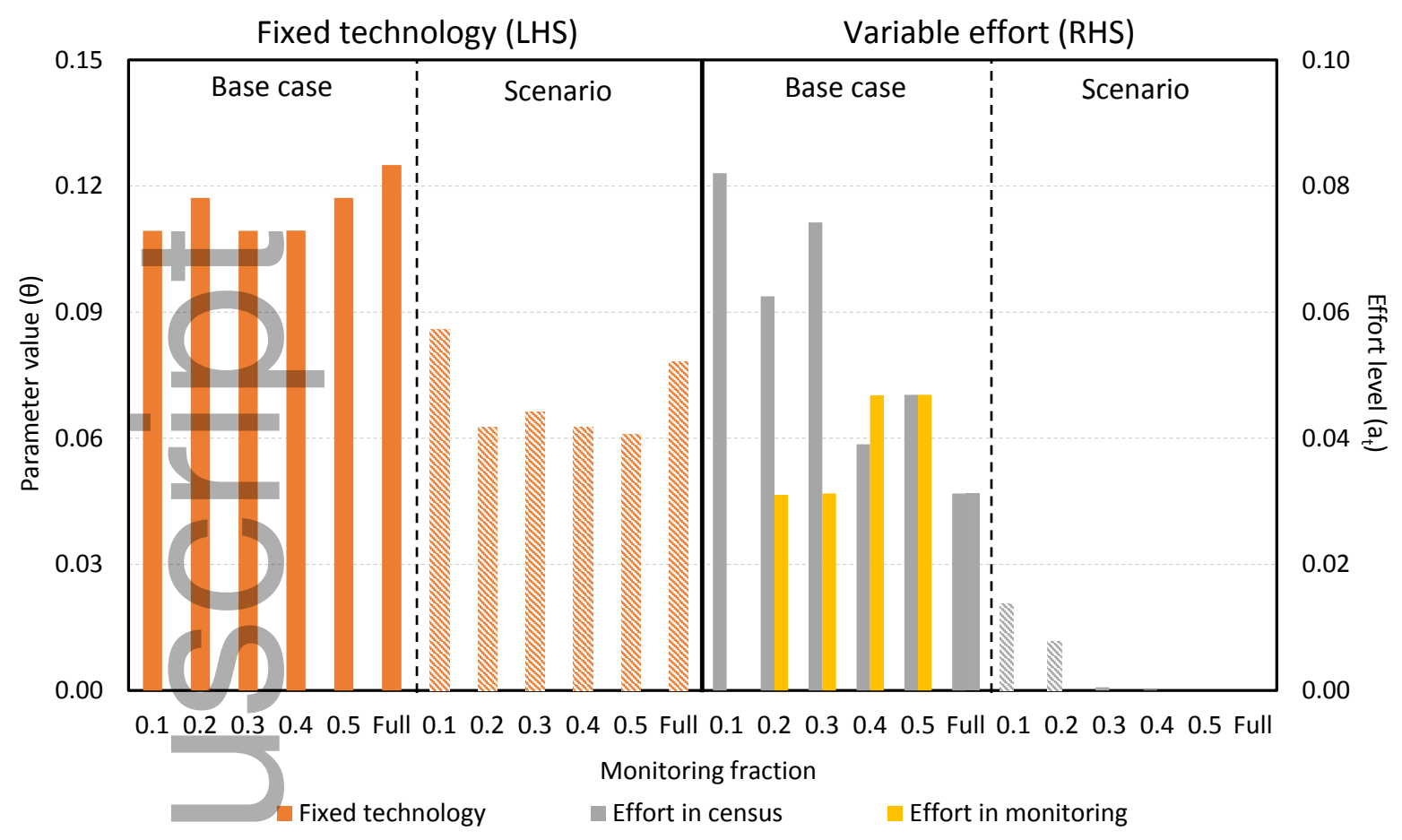

Figure 12: Optimal fixed technology $(\theta)$ and variable effort choices $\left(a_{t}\right)$ for the importer under the base case $(\lambda=25)$ and scenario where variable effort is less effective in reducing the approach rate $(\lambda=5)$. CSP-1 algorithm with a clearance number 10 and different monitoring fractions.

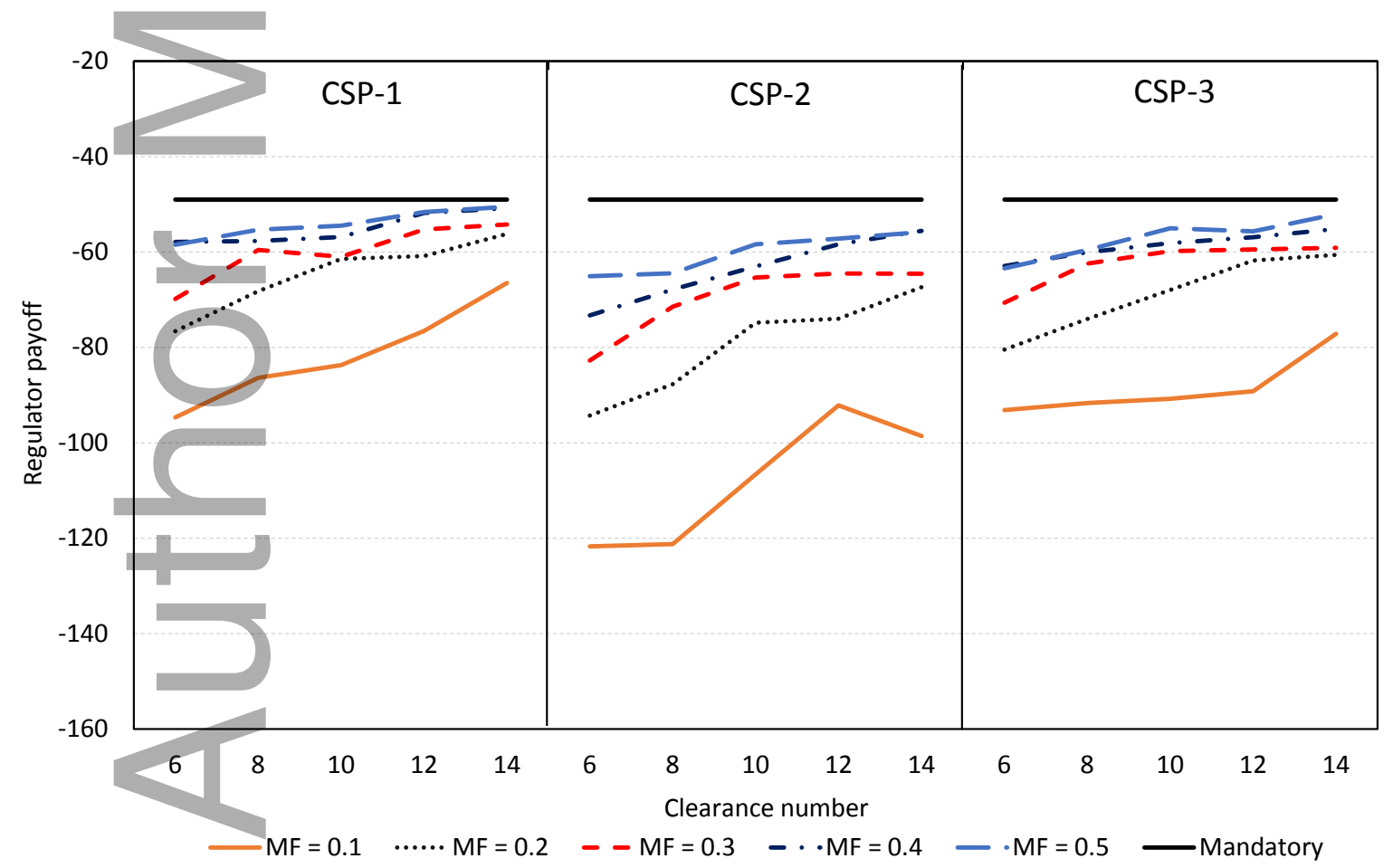

Figure 13: Regulator payoffs under the reduced effectiveness of variable effort $(\lambda=5)$ simulations for CSP-1, CSP-2 and CSP-3 rules. 


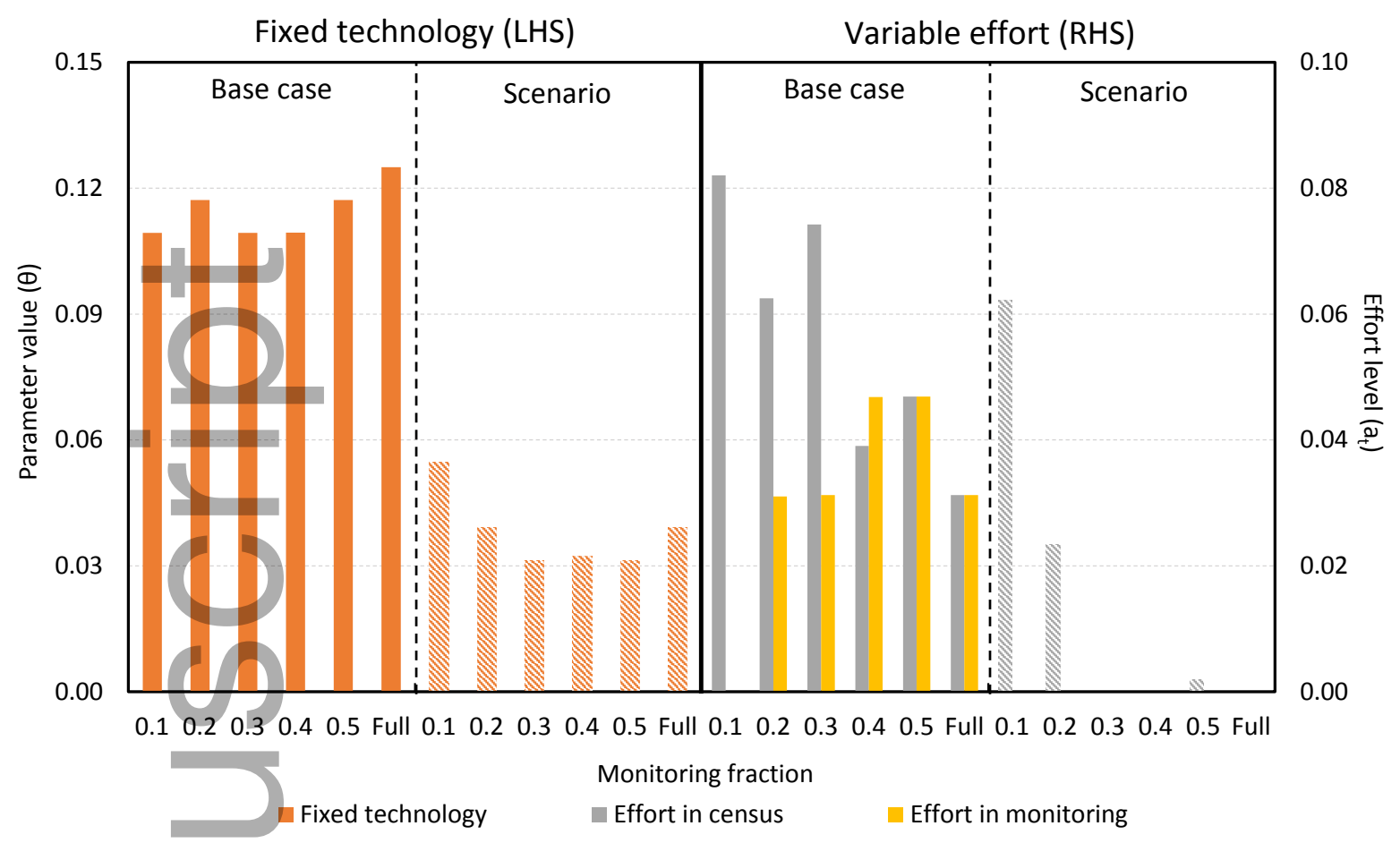

Figure 14: Optimal fixed technology $(\theta)$ and variable effort $\left(a_{t}\right)$ choices for the importer under the base case and low fixed technology cost scenario. CSP-1 algorithm with a clearance number 10 and different monitoring fractions.

are less dramatic in this situation, because the impact of "cheating" in monitoring mode is considerably reduced when low-cost fixed technologies are available. Our simulations suggest the CSP-3 rule with $C N=14$ and $M F=0.3$ delivered the highest expected payoff for the regulator in this scenario, though many $C N$ and $M F$ rule parameter values for the CSP-1 and CSP-3 rules in particular delivered higher payoffs than a mandatory inspection regime.

\section{iv Scenarios: importer inspection-related cost parameters}

The costs incurred by the importer in underdoing an inspection and treating contaminated consignments affect the penalty and reward structures inherent in the CSP algorithms. Based on the one-shot game in Section II, a higher cost of rectifying contaminated consignments should encourage the importer to reduce the biosecurity risk material approach rate. The one-shot game is less informative about the impact of the cost of being 


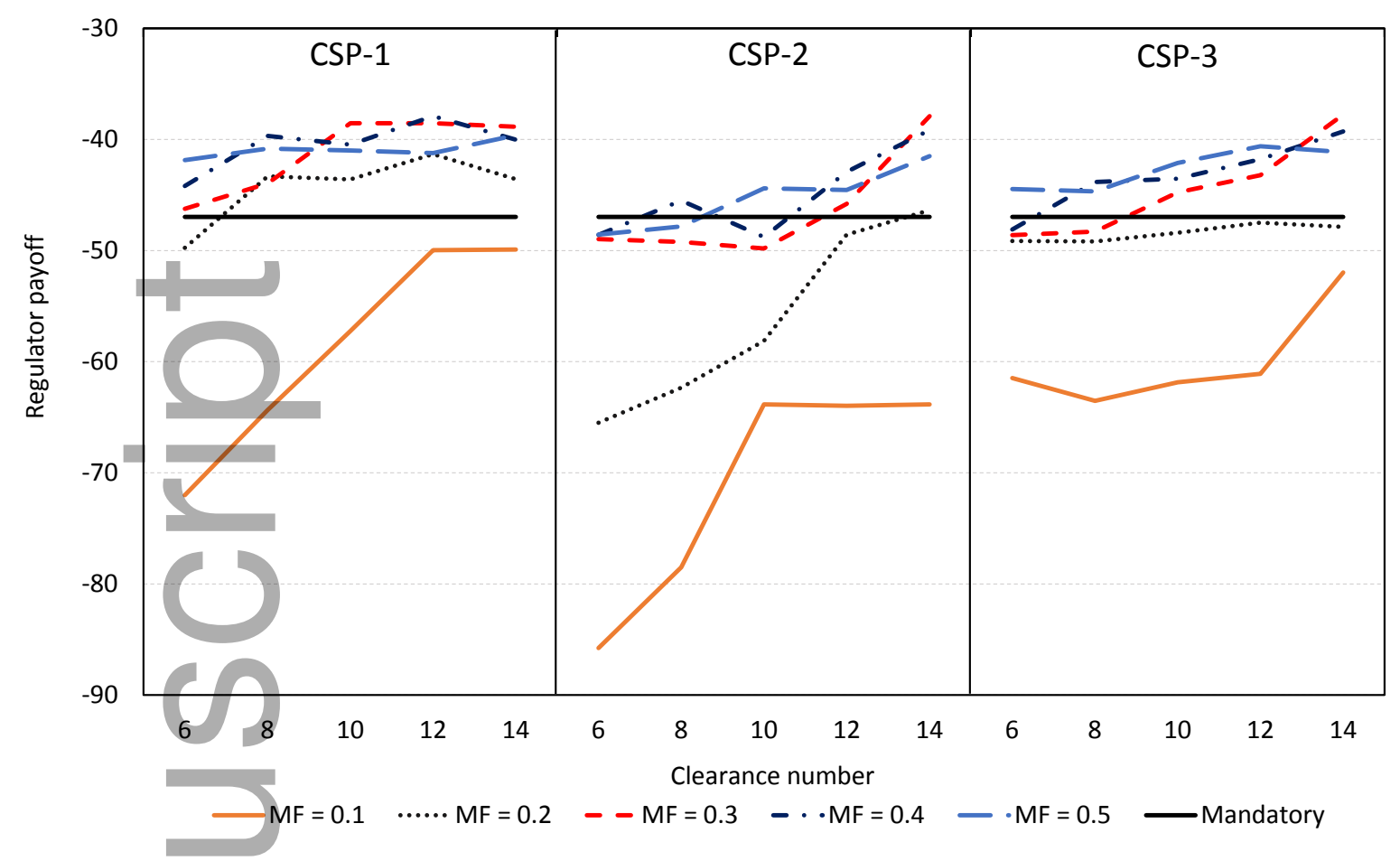

Figure 15: Regulator payoffs under the lower cost of fixed technology scenario simulations for CSP-1, CSP-2 and CSP-3 rules.

inspected, in part because it appears in a participation constraint that has a different influence in the repeated interaction setting. However, a lower cost of being inspected would be expected to amplify the importer's "cheating" behaviour.

Figure 16 compares the approach rates of biosecurity risk material under the different modes of the CSP-1 algorithm for the base case and two scenarios of interest, namely:

- where the cost to the importer of being inspected $(\omega)$ is lower; and

- where the importer faces a higher cost for rectifying contaminated consignments $(\kappa)$.

In the low inspection cost scenario, the approach rates of biosecurity risk material are generally higher in both the census and monitoring modes compared to the base case. This is consistent with the lower "benefit" received by the importer from avoiding inspections, which in turn reduces the return from undertaking abatement measures. For this set 


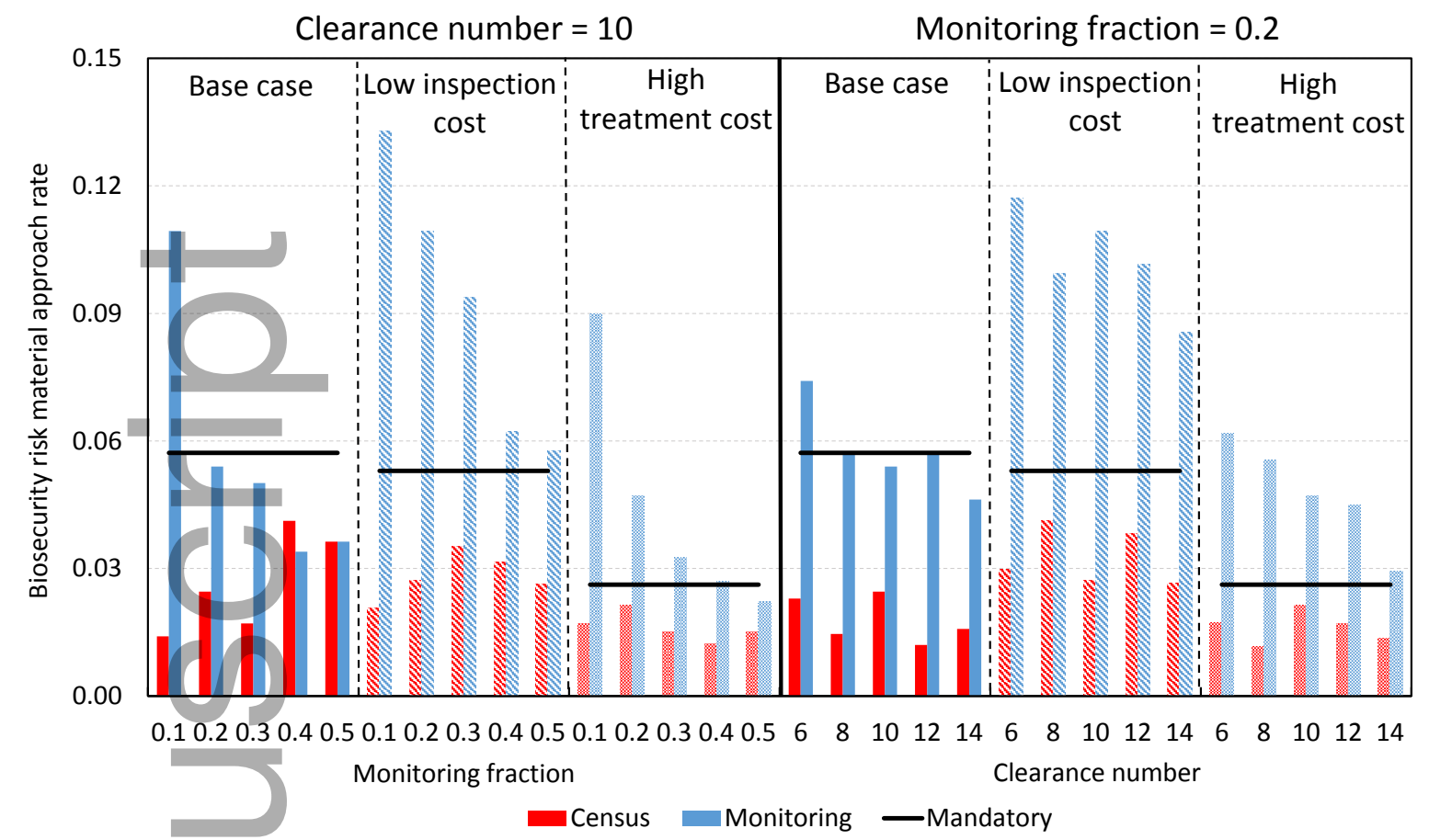

Figure 16: Probability of biosecurity risk material contamination based on importer's "optimal" response under the base case, the low inspection cost for the importer and high treatment cost scenarios. CSP-1 algorithm with different CSP rule parameters.

of parameters, the biosecurity regulator is better off adopting a mandatory inspection regime than a rule from the CSP family assessed in the grid search.

Consistent with the one-shot game, an importer facing a higher cost of treating contaminated consignments undertakes greater action to reduce the biosecurity risk material approach rate in both census and monitoring modes of the CSP-1 algorithm. The righthand panels in the two parts of Figure 16 also suggest the extent of "cheating" is lower in this scenario than the base case, reflecting the importer's self-driven incentive to reduce the likelihood of failing inspection and maintain a low approach rate. These actions result in the regulator having a wider range of CSP rule parameters where their payoff is higher than under a mandatory inspection mechanism (Figure 17), including some rules with the clearance number of 6 (the smallest used in our grid search) or a monitoring fraction as low as 0.2. According to the simulation analysis, the CSP-1 rule with parameters $C N=14$ and $M F=0.5$ offered the highest expected payoff to the regulator, providing 


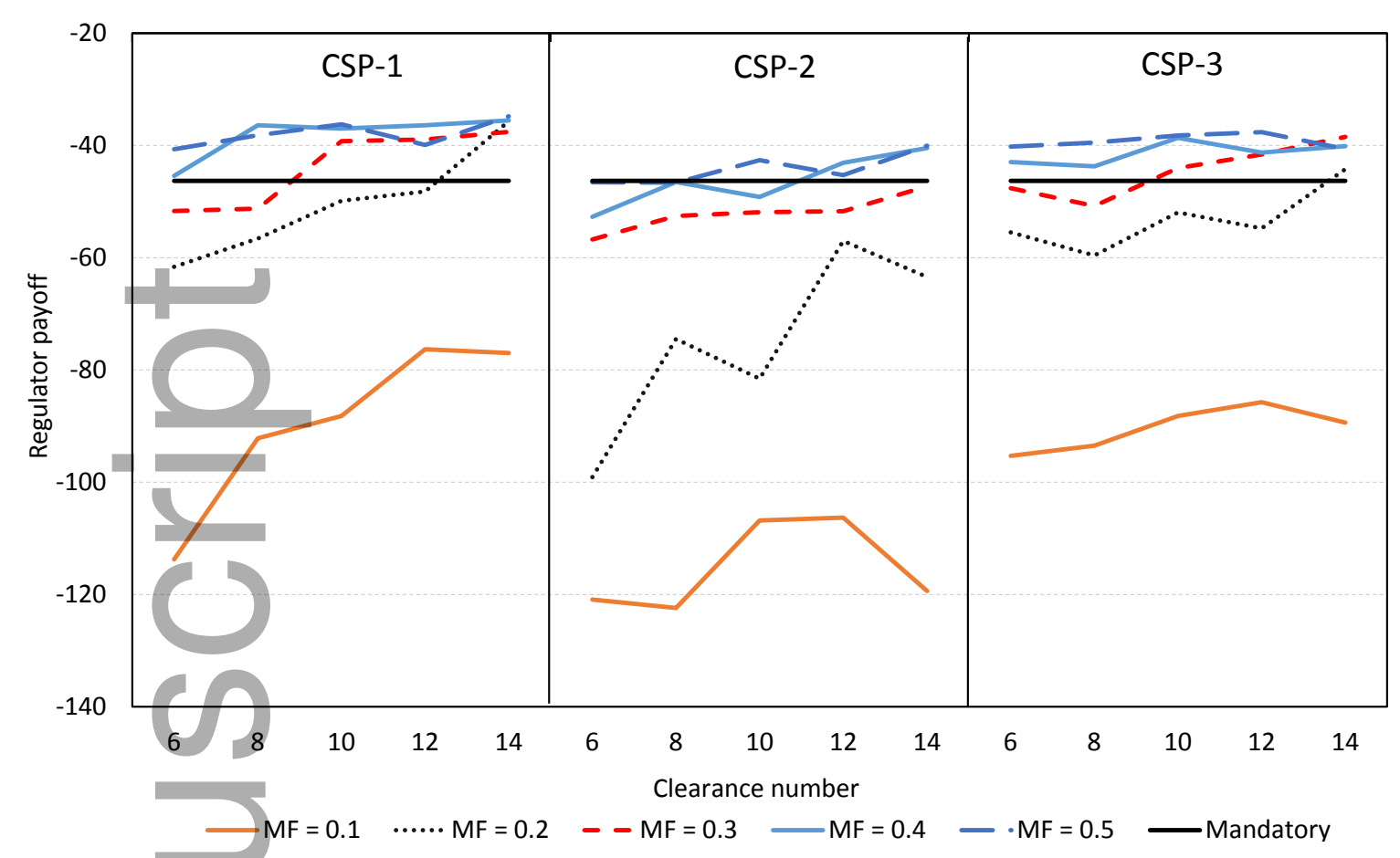

Figure 17: Regulator payoffs under the higher treatment cost scenario simulations for CSP-1, CSP-2 and CSP-3 rules.

close to a 25 per cent improvement on the expected payoff from a mandatory inspection regime.

\section{v Scenarios: regulator cost parameters and Type Two Error probabilities}

While the costs faced by the biosecurity regulator do not influence the importer's actions, they govern the regulator's trade-off between the disutility incurred by inspecting consignments and the consequences of failing to rectify consignments containing biosecurity risk material. In turn, this helps determine the rule parameters the biosecurity regulator is willing to choose from in meeting their regulatory objective.

Figure 18 compares the biosecurity regulator's payoff profiles for alternative parameterisations of the CSP-1 rule under the base case (left-hand panel) with two scenarios where the relative costs of the regulator undertaking an inspection and failing to rectify contam- 


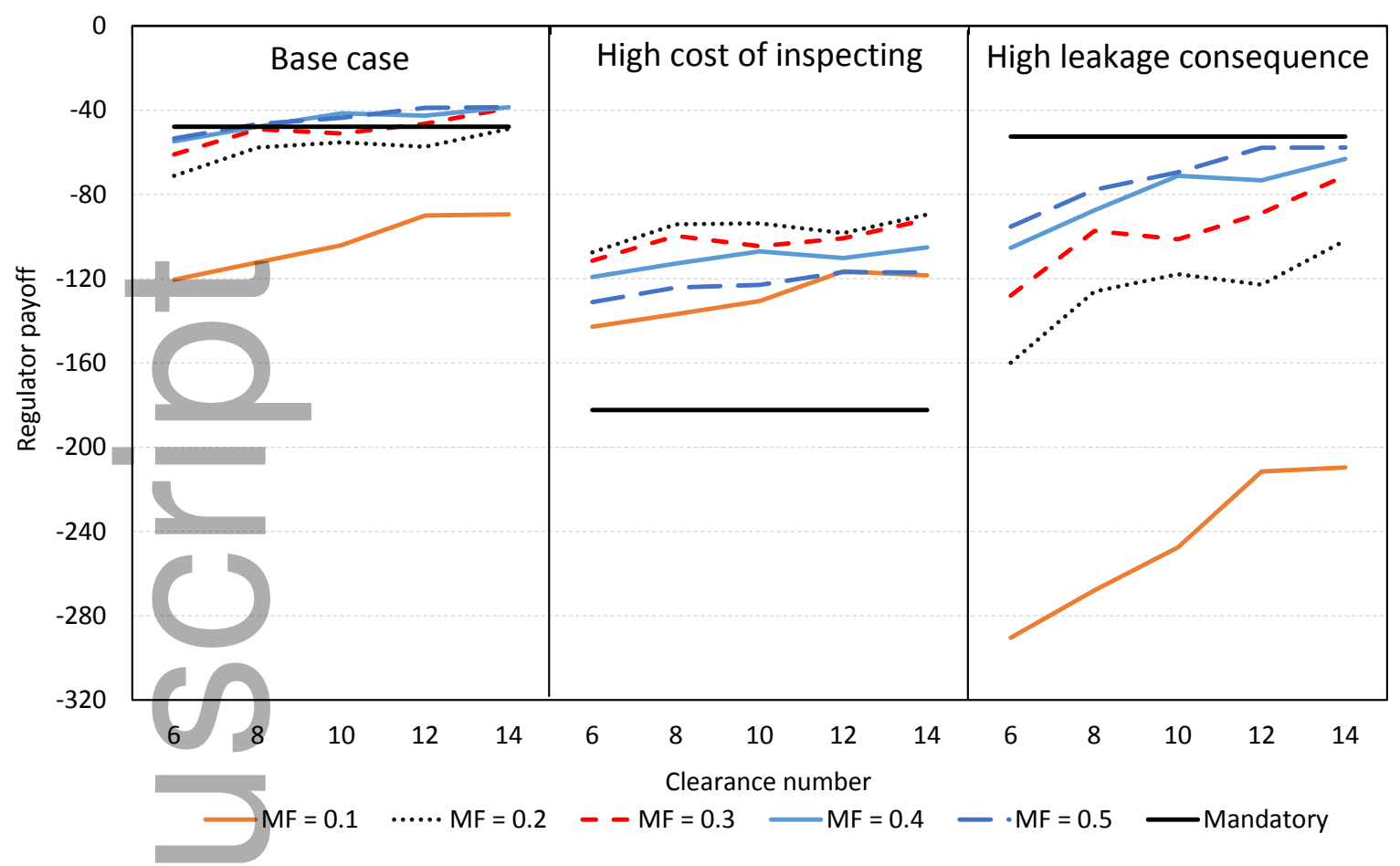

Figure 18: Regulator payoffs under the base case, high cost for the regulator of inspecting and high consequence of leakage for CSP-1 rules.

inated consignments differ. The middle panel illustrates a scenario where the regulator faces a higher implicit cost of inspecting consignments. A mandatory inspection regime is the regulator's least-preferred option here and they would rather implement a CSP rule with a low monitoring fraction and/or low clearance number. ${ }^{23}$ With a low relative consequences of leakage (the ratio $\frac{\gamma}{\chi}$ in the model) in this scenario, rules where the monitoring fraction is 0.2 or 0.3 are preferred to those at the extremes of the grid-search range. This is because an intermediate monitoring fraction does not expose the regulator to "cheating" by the importer to the same extent; an intermediate monitoring fraction also does not force the regulator to inspect too frequently without sufficient return. In contrast, the right-hand panel of Figure 18 shows the regulator will favour a mandatory inspection mechanism for goods with a high relative consequence of leakage. ${ }^{24}$

\footnotetext{
${ }^{23}$ The CSP-1 rule with $C N=14$ and $M F=0.2$ had the highest payoff for the regulator.

${ }^{24}$ Further investigation suggested that a mandatory inspection regime was optimal for the regulator in this parameterisation of the model if the ratio of the consequence of leakage to the cost of the regulator undertaking inspections $\left(\frac{\gamma}{\chi}\right)$ was around 48 or higher. In the right-hand panel of Figure $18, \frac{\gamma}{\chi}=60$.
} 
As suggested in Section II, a higher probability of failing to detect contaminated consignments encourages the importer to raise the approach rate in their consignments. This feature of importer behaviour is similar to reducing the treatment cost for contaminated consignments, as per the one-shot game, and means the regulator expects to incur a higher level of disutility based on the importer's choices. Relative to the base case, the optimality of different rule parameters changes slightly with the greater likelihood of inspection decision-errors, in that the CSP-1 rule with $C N=14$ and $M F=0.4$ provides the highest payoff to the regulator.

\section{Conchusion and policy implications}

In this paper, we have assessed how a biosecurity regulator can design border inspection rules to meet their objectives, taking into account the strategic response of a vertically integrated importer. This was illustrated through a one-shot inspection game, which we then extended to a dynamic setting where consignments are brought into the destination country over time. This allowed us to assess the suitability of inspection rules from the continuous sampling plan (CSP) family, where the probability that a given consignment is inspected depends on an importer's past compliance, that have been adopted by the DAWR.

Our results suggest that appropriately calibrated compliance-based inspection protocols can improve welfare in the importing country relative to a mandatory inspection regime. However, failing to calibrate rule parameters based on the importer's strategic response to the inspection rules they face can result in deleterious effects, as biosecurity risk material could leak into the local environment on a regular basis. The findings also reinforce that mandatory inspections may be the most appropriate inspection strategy for a biosecurity regulator to pursue for some types of imported products, based on the characteristics of the importer or the regulator's own preferences. 
The appropriateness of using CSP family rules on pathways depends on more than understanding the approach rate of biosecurity risk material under a mandatory inspection regime. Rather, the suitability of these rules depends on the production process for different goods, including the available methods to mitigate biosecurity risks, and the costs importers face in the inspection and rectification process. The costs a biosecurity regulator perceives in undertaking an inspection and addressing the leakage of biosecurity risk material also need to be taken into account, reflecting the explicit trade-off between reducing the rate of intervention and raising the potential for leakage.

Based on the results in this paper, inspection protocols based on the CSP rule family are likely to be most appropriate for the regulator to consider where:

- low-cost technologies are available to importers to reduce the likelihood of biosecurity risk material contamination. These technologies also need to be embedded in the production process to reduce the scope for "cheating" by importers undermining the regulatory regime;

- importers face significant costs in having to complete an inspection and/or rectify consignments found to have biosecurity risk material; and/or

- the consequences of biosecurity material leaking into the importing country for the regulator are low relative to their implicit cost of undertaking an inspection.

In practice, the biosecurity regulator cannot have complete knowledge of the characteristics about an importer that would affect how they respond to compliance-based inspection protocols. Furthermore, any type of imported product is likely to involve firms with a range of characteristics, resulting in a range of different responses to the same inspection protocol. While these information and multiplicity of "types" issues may not be able to be resolved completely, it may be possible to design a menu of inspection protocols from which importers could choose the option they believe would suit their circumstances best. If designed appropriately, these options could provide an incentive-compatible mechanism 
for firms to reveal information on their salient features that affect their "type" to the regulator.

While this paper has focused on the vertically integrated importer, many importers are independent operations and can choose from suppliers not connected with their operations. While several of the key principles addressed in this paper would carry over to a framework with supplier choice, the actions importers can take in response to the protocols will differ. Some of the factors that affect importer decision-making, such as information asymmetries between importers and suppliers over a supplier's approach rate and the costs importers face in switching suppliers, would also need to be accommodated.

Further theoretical work is warranted to explore the applicability of compliance-based inspection protocols under different supply-chain structures and the potential to use menus of protocols to distinguish between importers with differing abilities to reduce biosecurity risk material contamination. In addition, experimental studies are likely to be important to verify theoretical predictions around the effectiveness of these protocols and guide the roll-out of compliance-based inspection protocols as a regulatory mechanism for biosecurity risk management.

\section{References}

Abougamos, H., White, B. and Sadler, R. (2012). Contracts for grain biosecurity and grain quality. Contributed paper prepared for presentation at the 56th Australian Agricultural and Resource Economics Society Conference, Fremantle.

Andreozzi, L. (2004). Rewarding policemen increases crime. another surprising result from the inspection game., Public Choice 121(1-2): 69-82.

Anscombe, F. J. (1958). Rectifying inspection of a continuous output, Journal of the American Statistical Association 53(283): 702-719. 
Australian Government Department of Finance (2014). Australian Government Cost Recovery Guidelines, third edn. Resource Management Guide No. 304.

Avenhaus, R. (2004). Applications of inspection games, Mathematical Modelling and Analysis 9(3): 179-192.

Avenhaus, R. and Krieger, T. (2011). Unannounced interim inspections: Do false alarms matter?, Mathematical Modelling and Analysis 16(1): 109-118.

Avenhaus, R., Okada, A. and Zamir, S. (1991). Inspector leadership with incomplete information, in R. Selten (ed.), Game Equilibrium Models IV: Social and Political Interaction, Springer Verlag, Berlin, pp. 319-361.

Avenhaus, R., von Stengel, B. and Zamir, S. (2002). Inspection games, in R. J. Aumann and S. Hart (eds), Handbook of Game Theory with Economic Applications, Vol. 3, North-Holland, Amsterdam, pp. 1947-1987.

Bakır, N. O. (2011). A Stackelberg game model for resource allocation in cargo container security, Annals of Operations Research 187(1): 5-22.

Barr, M. S., Mullainathan, S. and Shafir, E. (2013). Behaviorally informed regulation, in E. Shafir (ed.), The Behavioral Foundations of Public Policy, Princeton University Press, Princeton, pp. 440-461.

Beale, R., Fairbrother, J., Inglish, A. and Trebeck, D. (2008). One Biosecurity: a working partnership, Commonwealth of Australia, Canberra.

Berentsen, A., Bruegger, E. and Loertscher, S. (2008). On cheating, doping and whisteblowing, European Journal of Political Economy 24(2): 415-436.

Besancenot, D. and Vranceanu, R. (2007). Equilibrium (dis)honesty, Journal of Economic Behavior and Organization 64(2): 232-249.

Buetre, B., Wicks, S., Kruger, H., Millist, N., Yainshet, A., Garner, G., Duncan, A., Abdalla, A., Trestrail, C., Hatt, M., Thompson, L.-J. and Symes, M. (2013). Po- 
tential socio-economic impacts of an outbreak of foot-and-mouth disease in Australia, ABARES Research Report 13.11, Canberra.

Case, K. E., Bennett, G. K. and Schmidt, J. W. (1973). The Dodge CSP-1 continuous sampling plan under inspection error, AIIE Transactions 5(3): 193-202.

Chou, P. B., Wu, W., Anandarajan, A. and Valenti, D. (2012). Is honesty the best policy? a game theory perspective of auditing, International Journal of Behavioural Accounting and Finance 3(1/2): 88-106.

Chun, Y. H. (2010). Bayesian inspection model for the production process subject to a random failure, IIE Transactions 42(4): 304-316.

Commonwealth of Australia (2014). Communique - National Management Group endorses National Banana Freckle Response Plan. Available from http://www.agriculture.gov.au/about/media-centre/communiques/nmg-bananafreckle-oct-14.

Commonwealth of Australia (2015). Agricultural Competitiveness White Paper. Canberra.

Commonwealth of Australia (2016). Budget 2016-17: Portfolio Budget Statements 201617, Budget Related Paper 1.1: Agriculture and Water Resources Portfolio, Canberra.

Delle Fave, F. M., Jiang, A. X., Yin, Z., Zhang, C., Tambe, M., Kraus, S. and Sullivan, J. P. (2014). Game-theoretic security patrolling with dynamic execution uncertainty and a case study on a real transit system, Journal of Artificial Intelligence Research 50: $321-367$.

Dodge, H. F. (1943). A sampling inspection plan for continuous production, The Annals of Mathematical Statistics 14(3): 264-279.

Dodge, H. F. and Torrey, M. N. (1951). Additional continuous sampling inspection regimes, Industrial Quality Control 7(5): 7-12. 
Fernandez, L. and Sheriff, G. (2013). Optimal border policies for invasive species under asymmetric information, Environmental and Resource Economics 56(1): 27-45.

Franckx, L. (2002). The use of ambient inspections in environmental monitoring and enforcement when the inspection agency cannot commit itself to announced inspection probabilities, Journal of Environmental Economics and Management 43(1): 71-92.

Friesen,L. (2003). Targeting enforcement to improve compliance with environmental regulations, Journal of Environmental Economics and Management 46(1): 72-85.

Friesen, L. (2006). The social welfare implications of industry self-auditing, Journal of Environmental Economics and Management 51(3): 280-294.

Hafi, A., Addai, D., Zhang, K. and Gray, E. M. (2015). The value of Australia's biosecurity system at the farm gate: An analysis of avoided trade and on-farm impacts, ABARES Research Report 15.2, Department of Agriculture, Canberra.

Hennessy, D. A. (2008). Biosecurity incentives, network effects, and entry of a rapidly spreading pest, Ecological Economics 68(1-2): 230-239.

Hillier, F. S. (1964). New criteria for selecting continuous sampling plans, Technometrics 6(2): 161-178.

Hohzaki, R. (2011). An inspection game with smuggler's decision about the amount of contraband, Journal of the Operations Research Society of Japan 54(1): 25-45.

Horan, R. D. and Lupi, F. (2005). Tradeable risk permits to prevent future introductions of invasive alien species into the Great Lakes, Ecological Economics 52(3): 289-304.

James, S. and Anderson, K. (1998). On the need for more economic assessment of quarantine policies, The Australian Journal of Agricultural and Resource Economics $42(4): 425-444$.

Jin, G. Z. and Lee, J. (2011). The imperfection of human inspectors: Lessons from Florida restaurant inspections. unpublished manuscript, 17 March. 
Kilgour, D. M. (1994). The use of costless inspection in enforcement, Theory and Decision 36(3): 207-232.

Kirstein, R. (2014). Doping, the inspection game, and Bayesian enforcement, Journal of Sports Economics 15(4): 385-409.

Kobyashi, M. and Melkonyan, T. (2011). Strategic incentives in biosecurity actions: Theoretical and empirical analyses, Journal of Agricultural and Resource Economics 36(2): $242-262$.

Krawczyk, M. (2009). The role of repetition and observability in deterring insurance fraud, The Geneva Risk and Insurance Review 34(1): 74-87.

Krieger, T. (2011). A generalization of Diamond's inspection model: Errors of the first and second kind, in B. Hu, K. Morasch, S. Pickl and M. Siegle (eds), Operations Research Proceedings 2010, Springer-Verlag, Berlin, pp. 77-82.

McAusland, C. and Costello, C. (2004). Avoiding invasives: trade-related policies for controlling unintentional exotic species introductions, Journal of Environmental Economics and Management 48(2): 954-977.

Munford, A. G. (1981). Comparison among certain inspection policies, Management Science 27(3): 260-267.

Plant Health Australia (2011). Plan for transition to management of Myrtle rust. Available from http://myrtlerust.net.au/wordpress/wpcontent/uploads/2011/11/Transition-to-Management-Plan-v1-Nov-2011.pdf.

Pradiptyo,- R. (2007). Does punishment matter? a refinement of the inspection game, Review of Law and Economics 3(2): 197-219.

Robinson, A., Bell, J., Woolcott, B. and Perotti, E. (2012). AQIS quarantine operations risk return: Imported plant-product pathways, ACERA 1001 Study J: Final report, 1 June. 
Rossiter, A., Hester, S., Aston, C., Sibley, J., Stoneham, G. and Woodhams, F. (2016). CEBRA Project 1304C: Incentives for importer choices, Final report. Available from: http://cebra.unimelb.edu.au/publications/reports.

Rothenstein, D. and Zamir, S. (2002). Imperfect inspection games over time, Annals of Operations Research 109: 175-192.

Springborn, M. R. (2014). Risk aversion and adaptive management: Insights from a multiarmed bandit model of invasive species risk, Journal of Environmental Economics and Management 68(2): 226-242.

Surkov, I. V., Oude Lansink, A. G. J. M., van Kooten, O. and van der Werf, W. (2008). A model of optimal import phytosanitary inspection under capacity constraint, Agricultural Economics 38(3): 363-373.

World Trade Organisation (n.d.). Agreement on the Application of Sanitary and Phytosanitary Measures. Opened for signature 15 April 1994, 1867 UNTS 493 (entered into force 1 January 1995), available at http://www.wto.org/ (SPS Agreement). 
Regulator announces probability she will inspect consignment $(0 \leq \beta \leq 1)$

Importer chooses effort to put into mitigating biosecurity risk $(a \geq 0)$

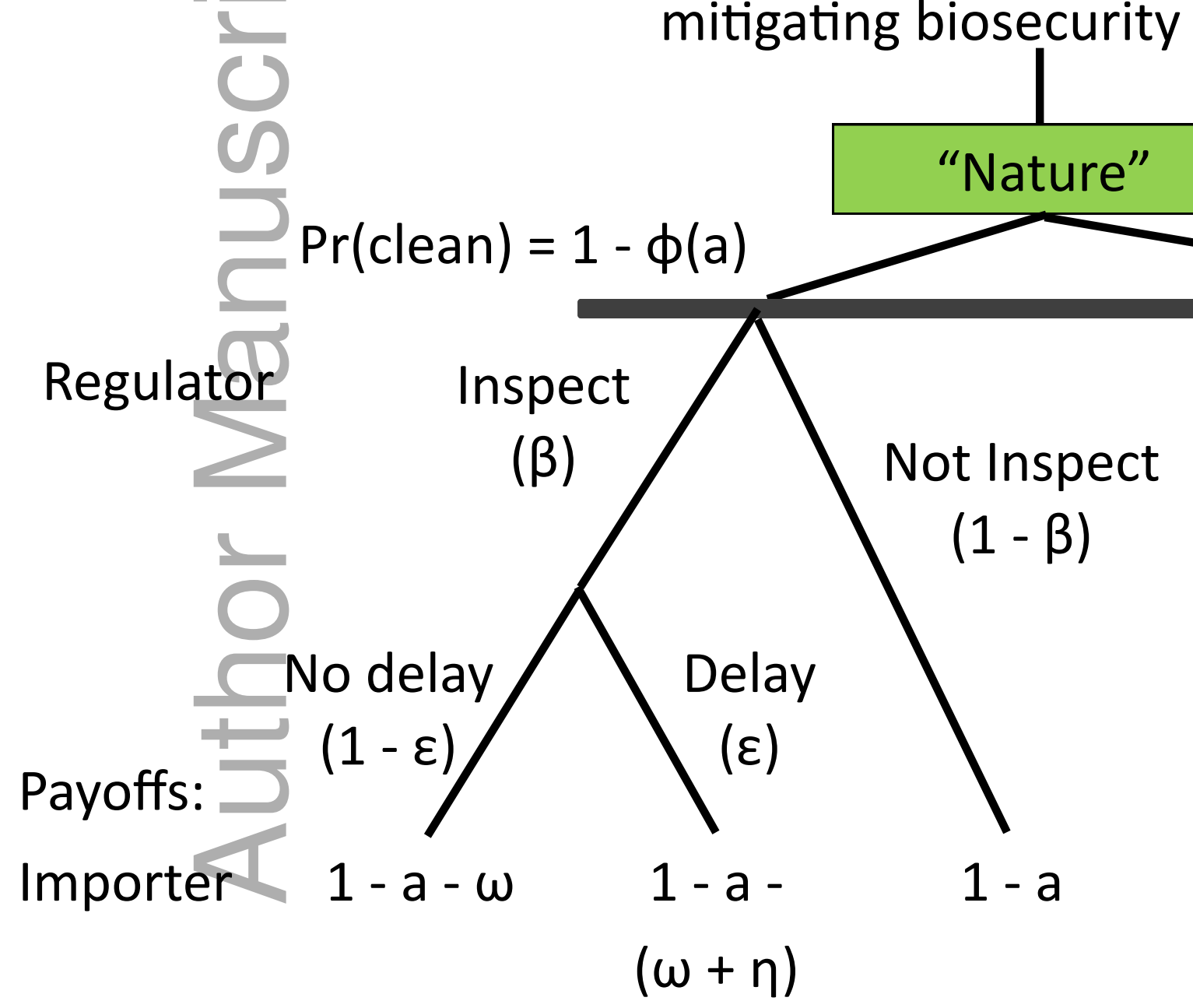

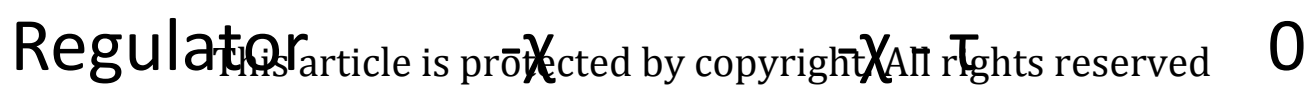

$-x \quad-x-y$

$-x \quad-x-y$

$-\gamma$

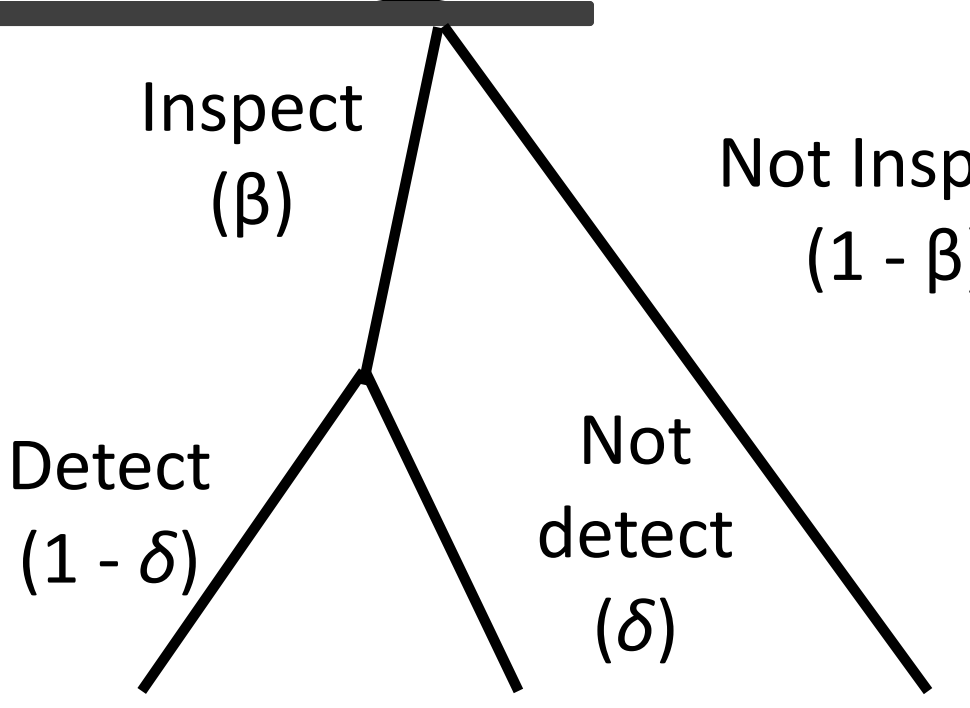

$1-a-$

$1-a-\omega$

$1-a$

$(\omega+k)$ 


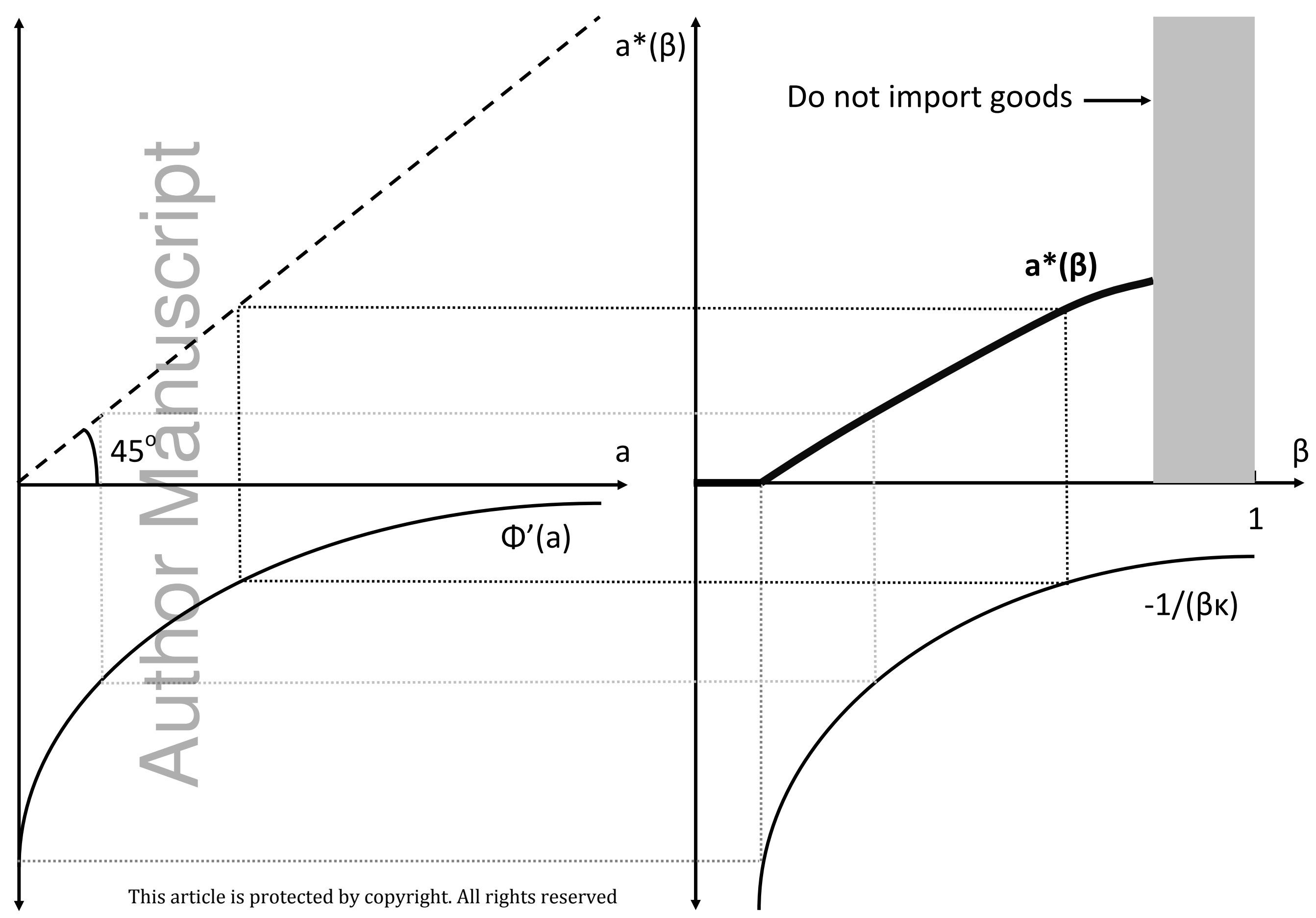


ecor_12315_f3.pdf

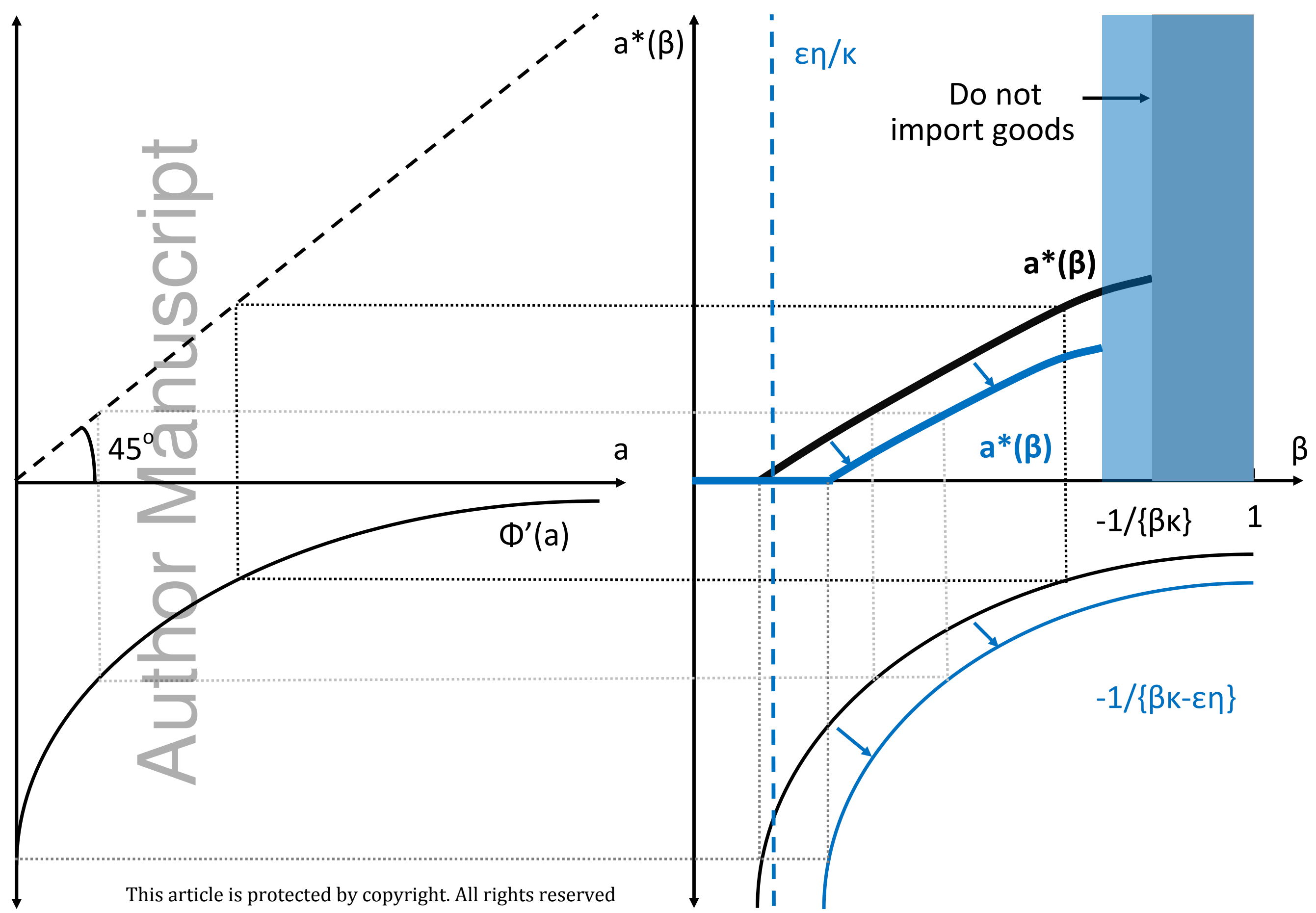




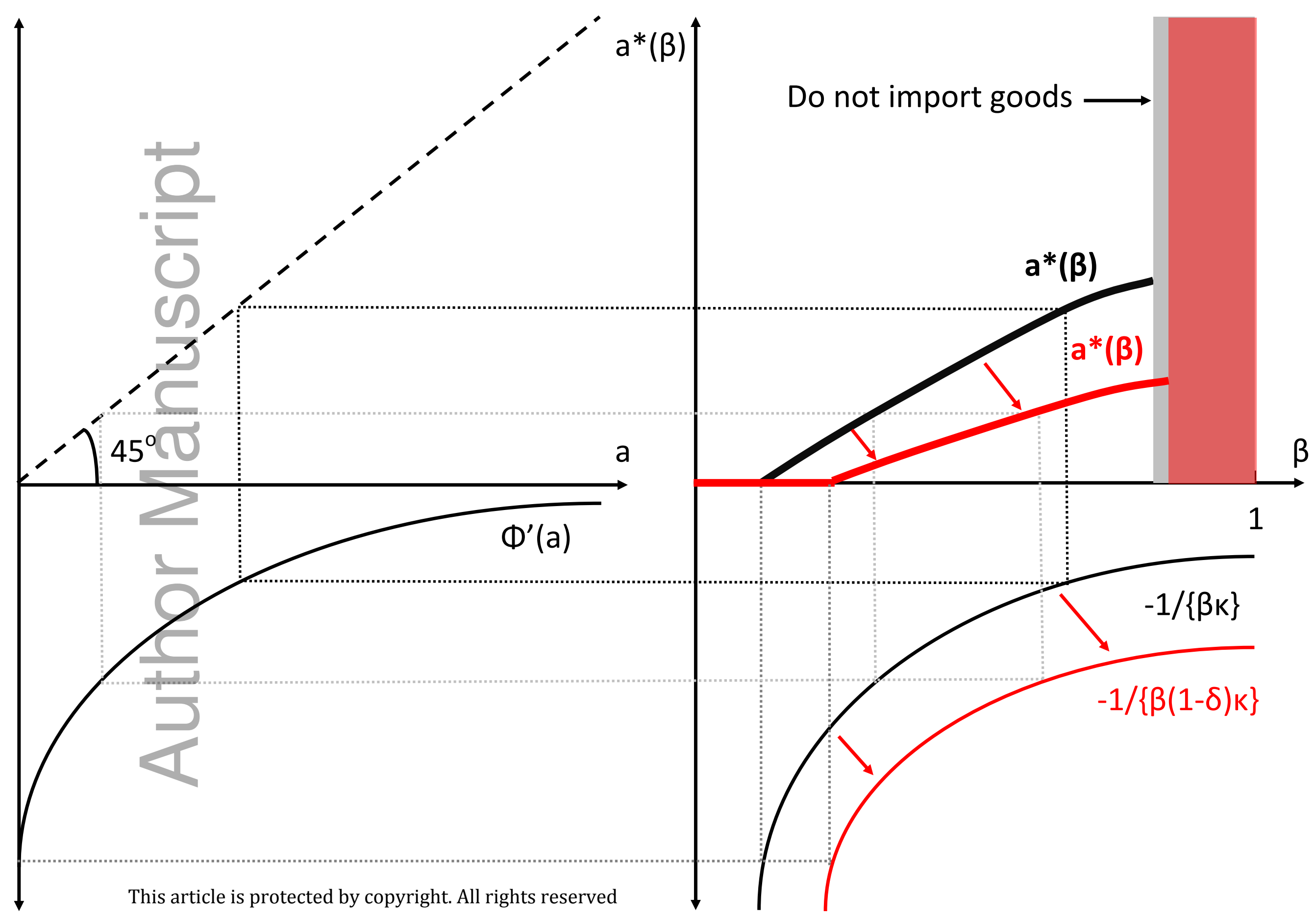




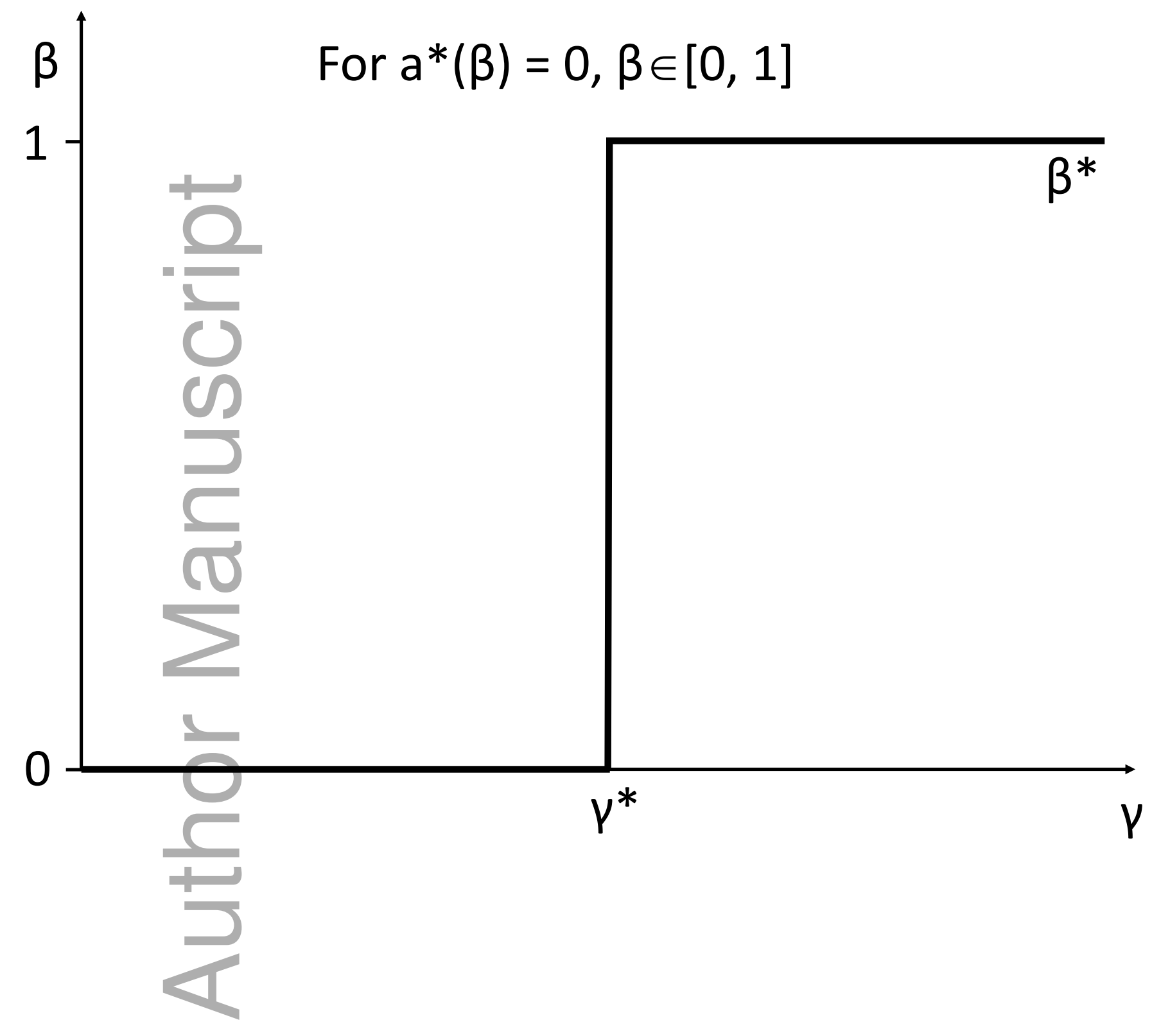




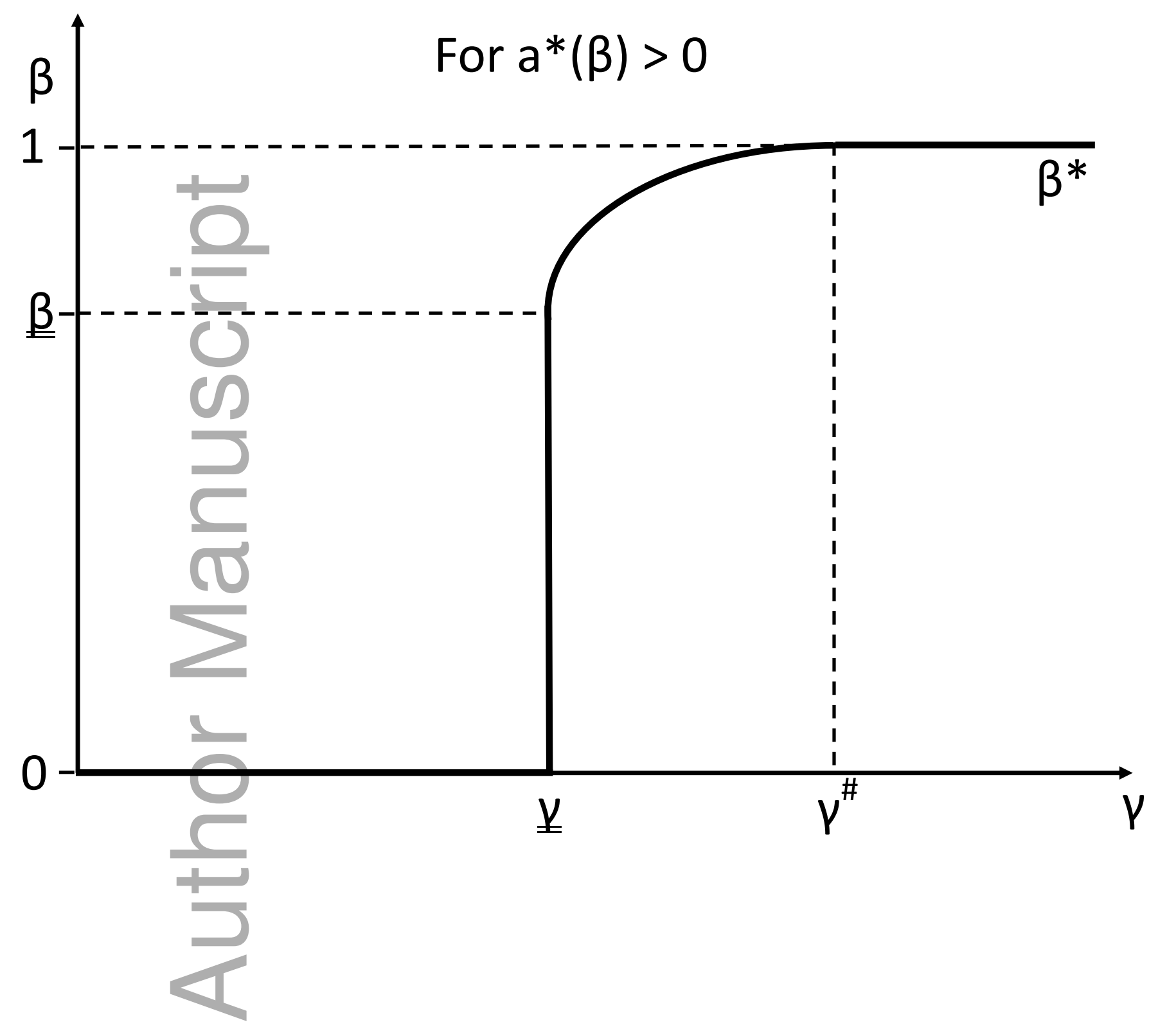


If áconsignment fails inspection

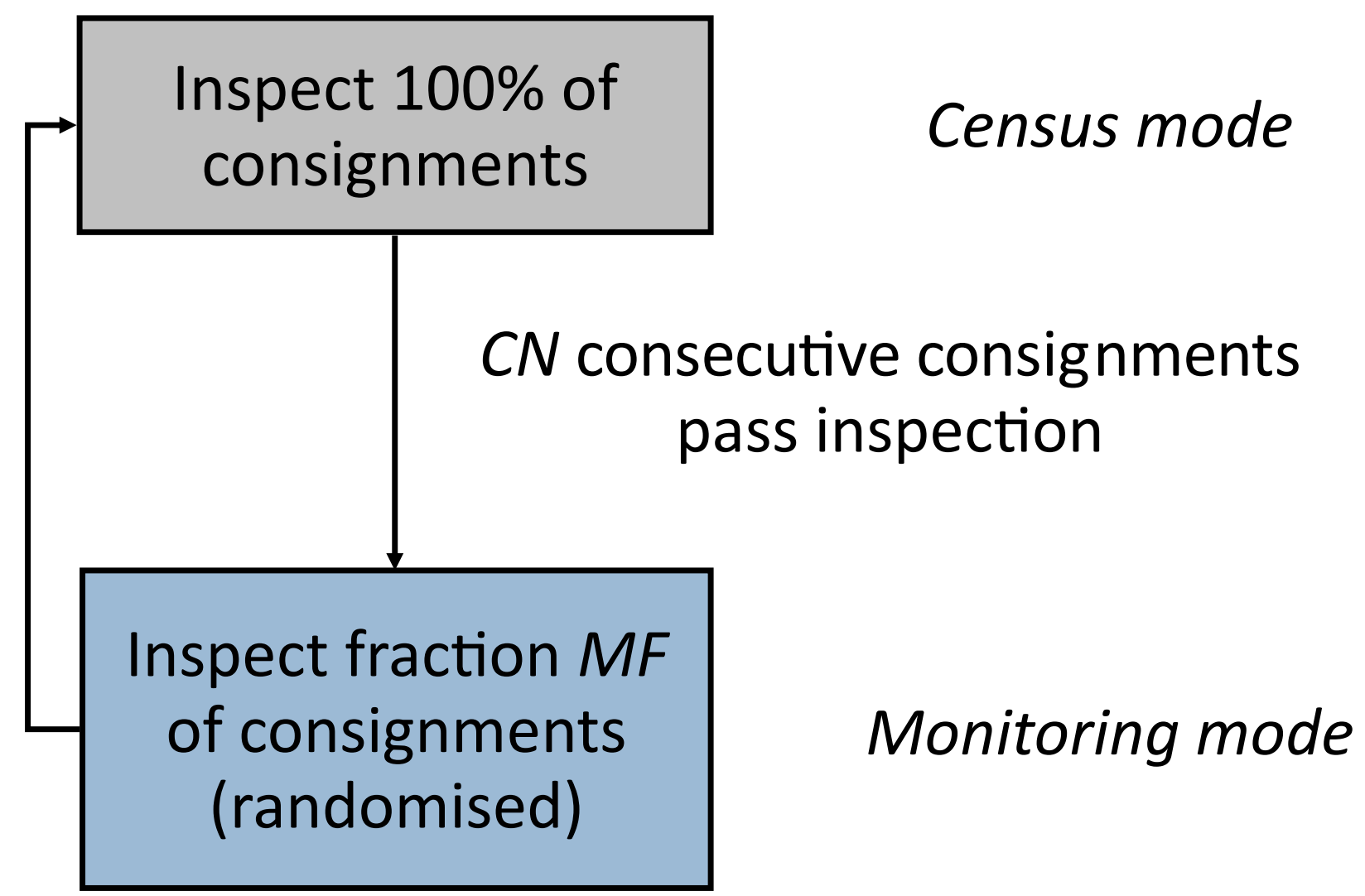




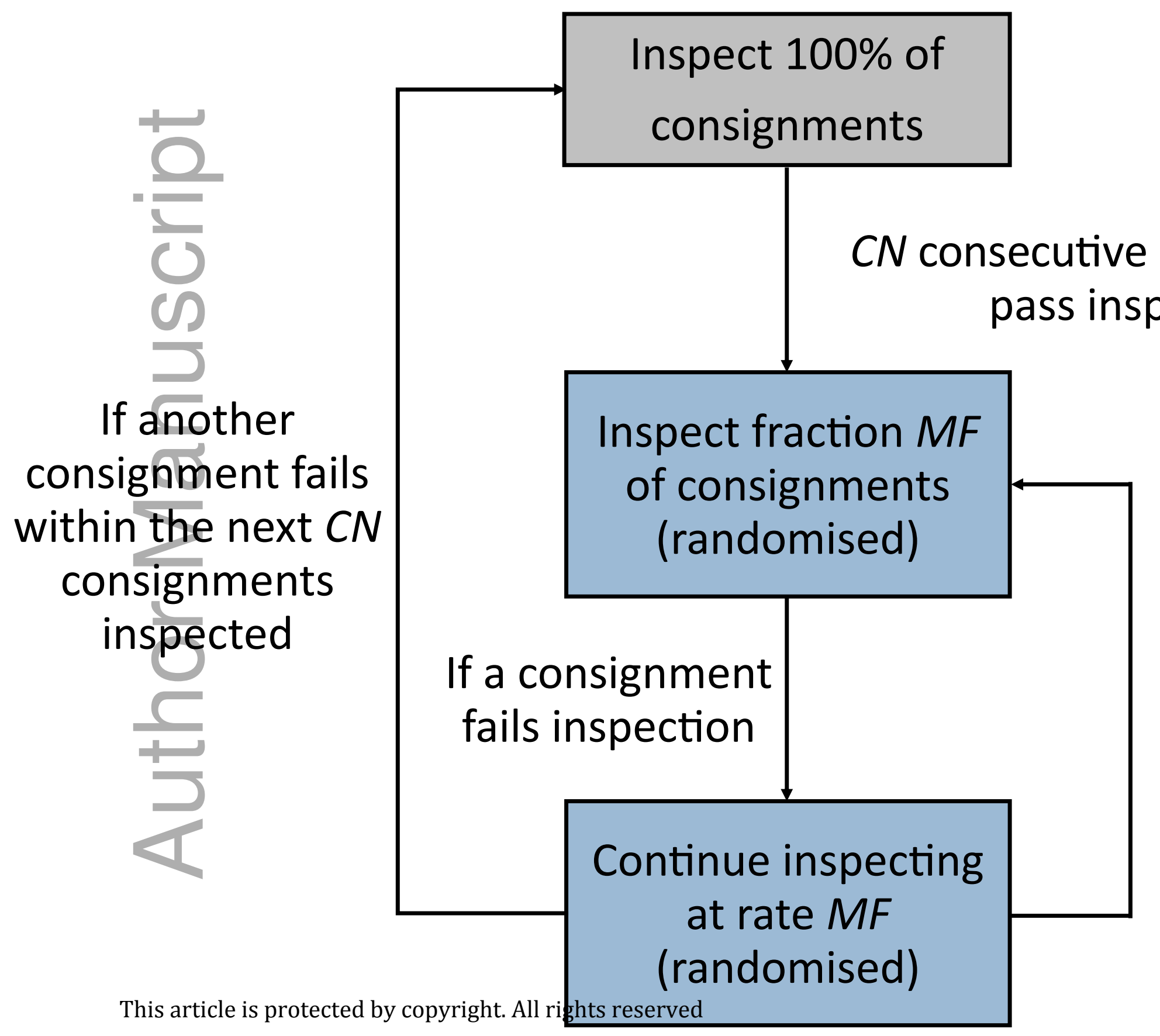

Census mode

Monitoring mode

$C N$ consecutive consignments pass inspection

Failure detection mode 


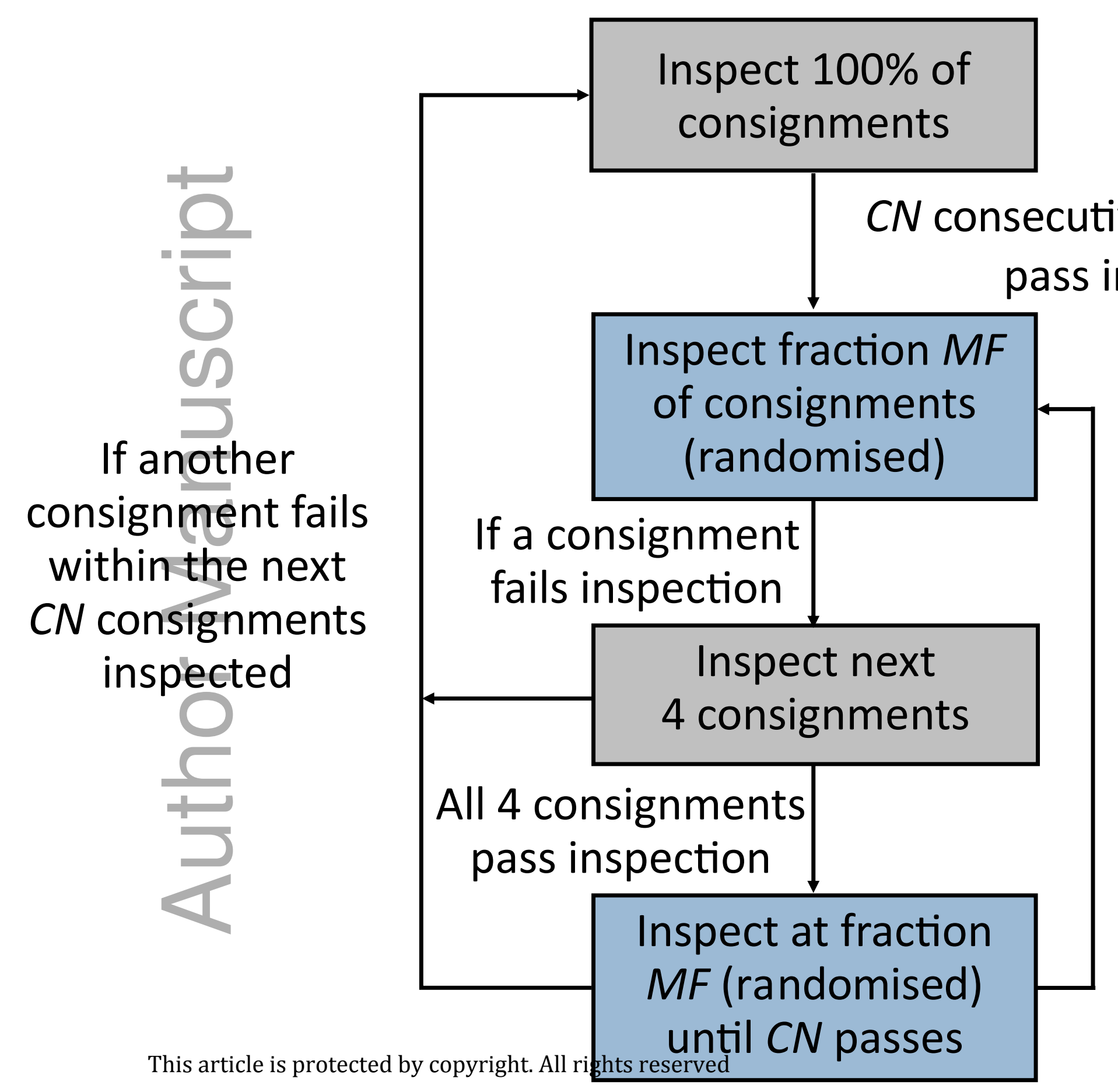

\section{Census mode}

Monitoring mode

$\mathrm{CN}$ consignments pass inspection in a row

Tight census mode

Failure detection mode 


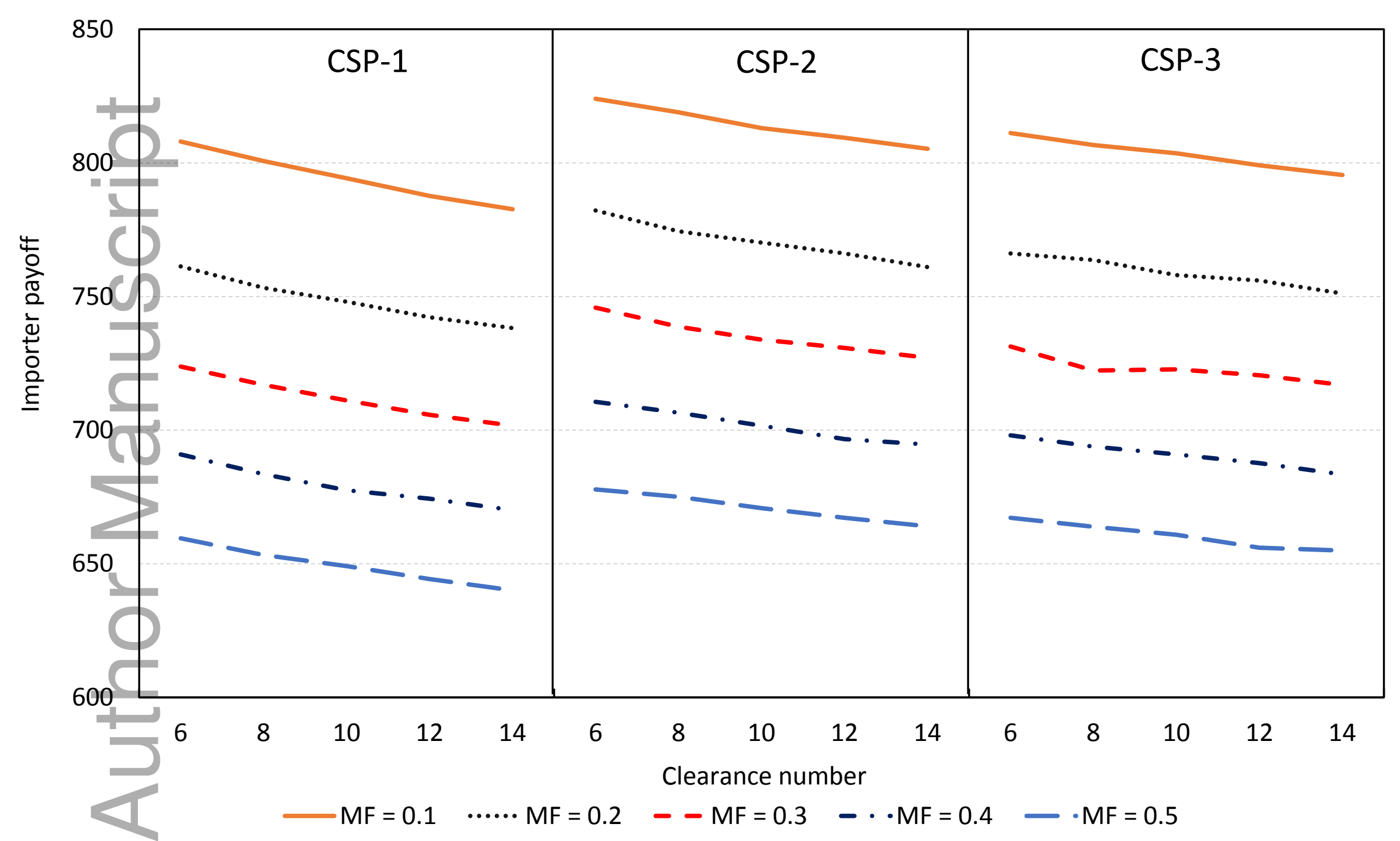




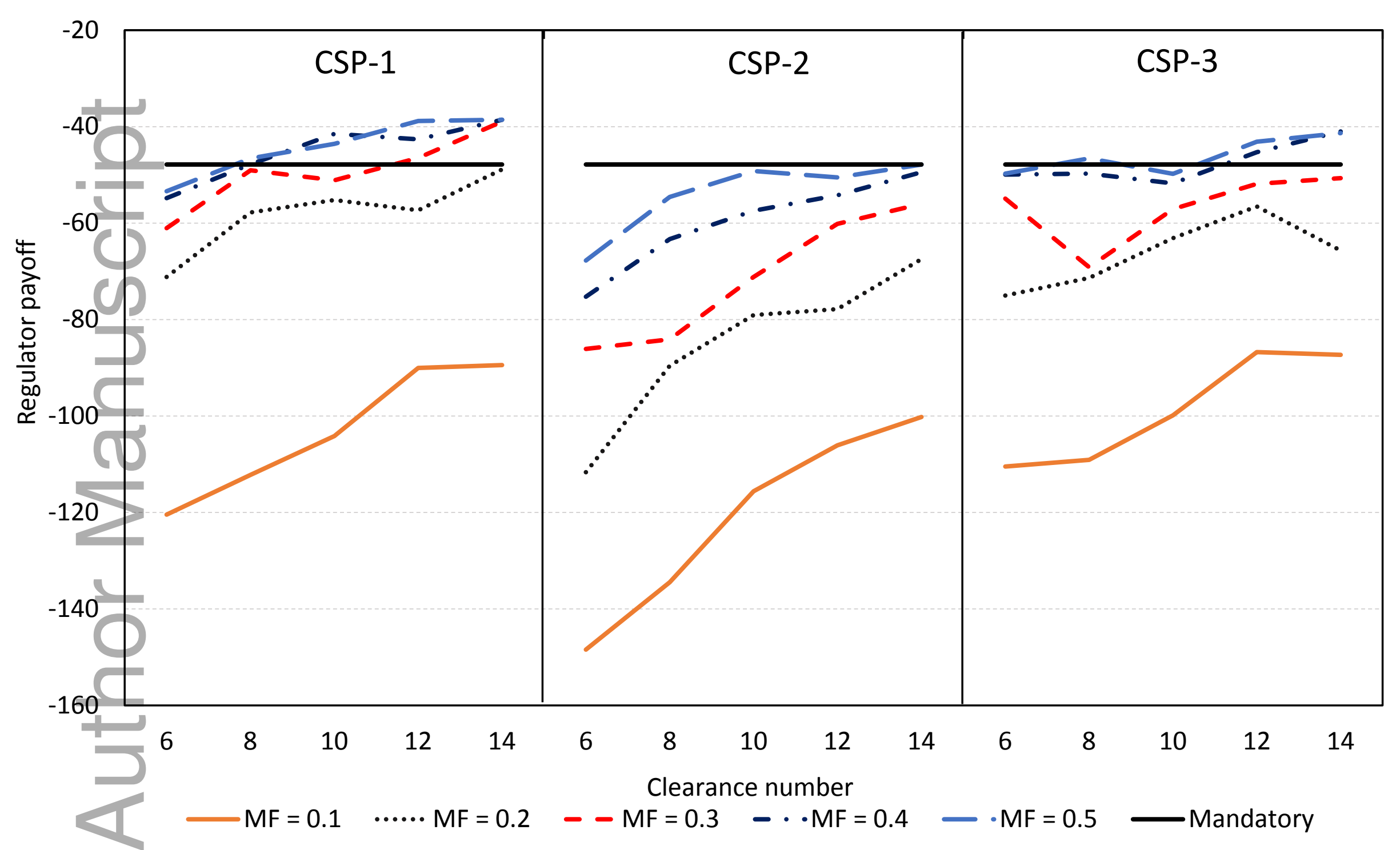

This article is protected by copyright. All rights reserved 


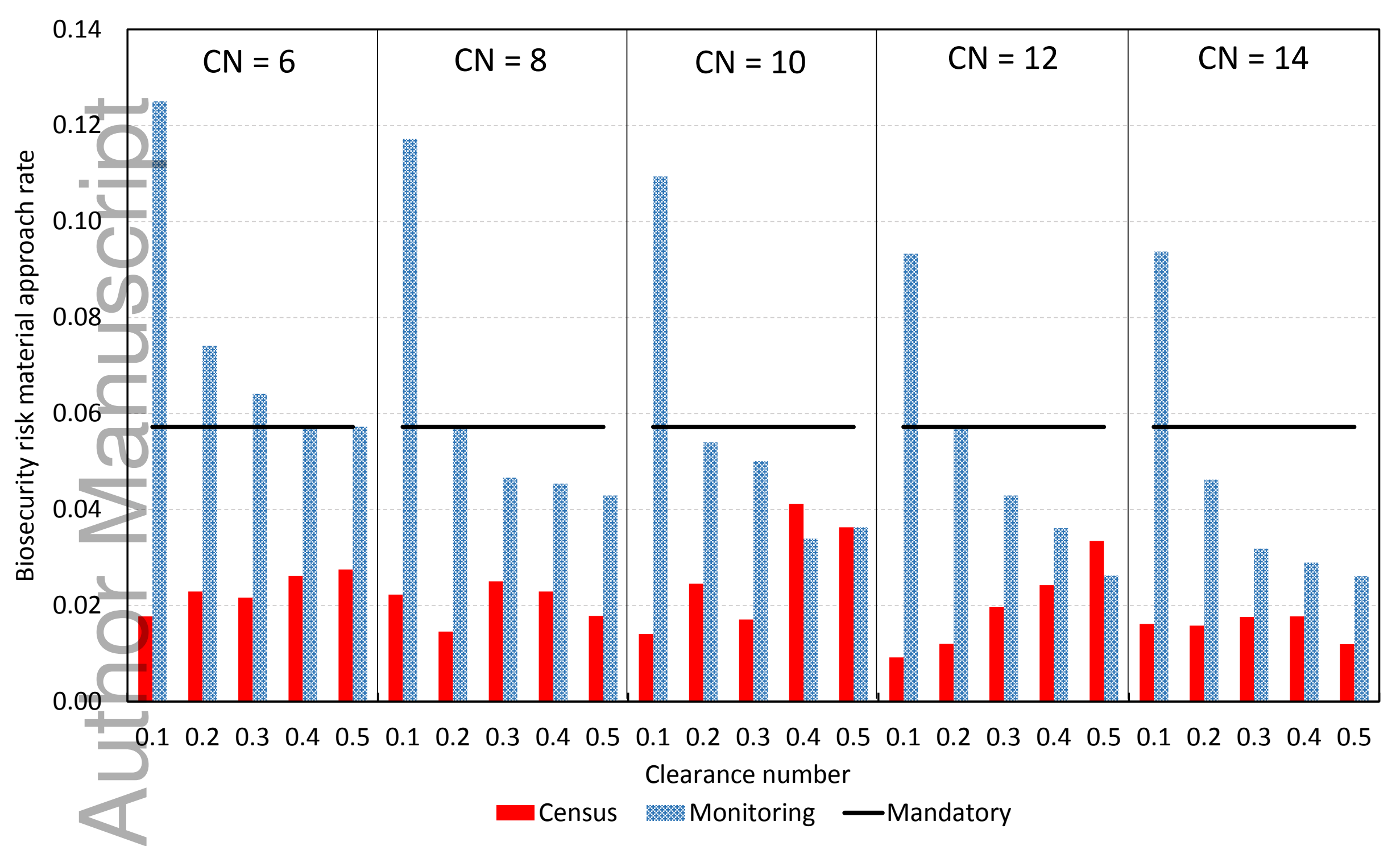




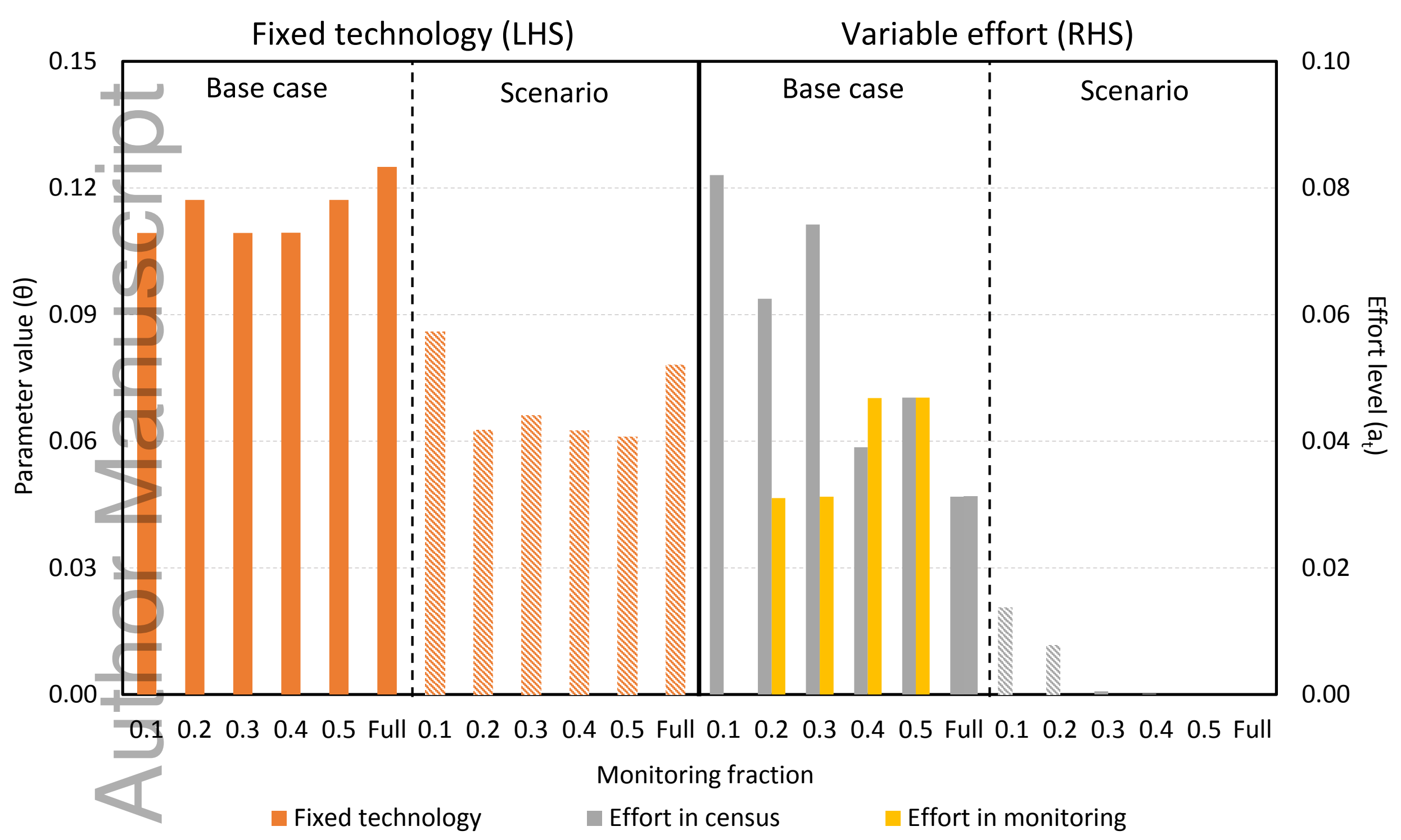




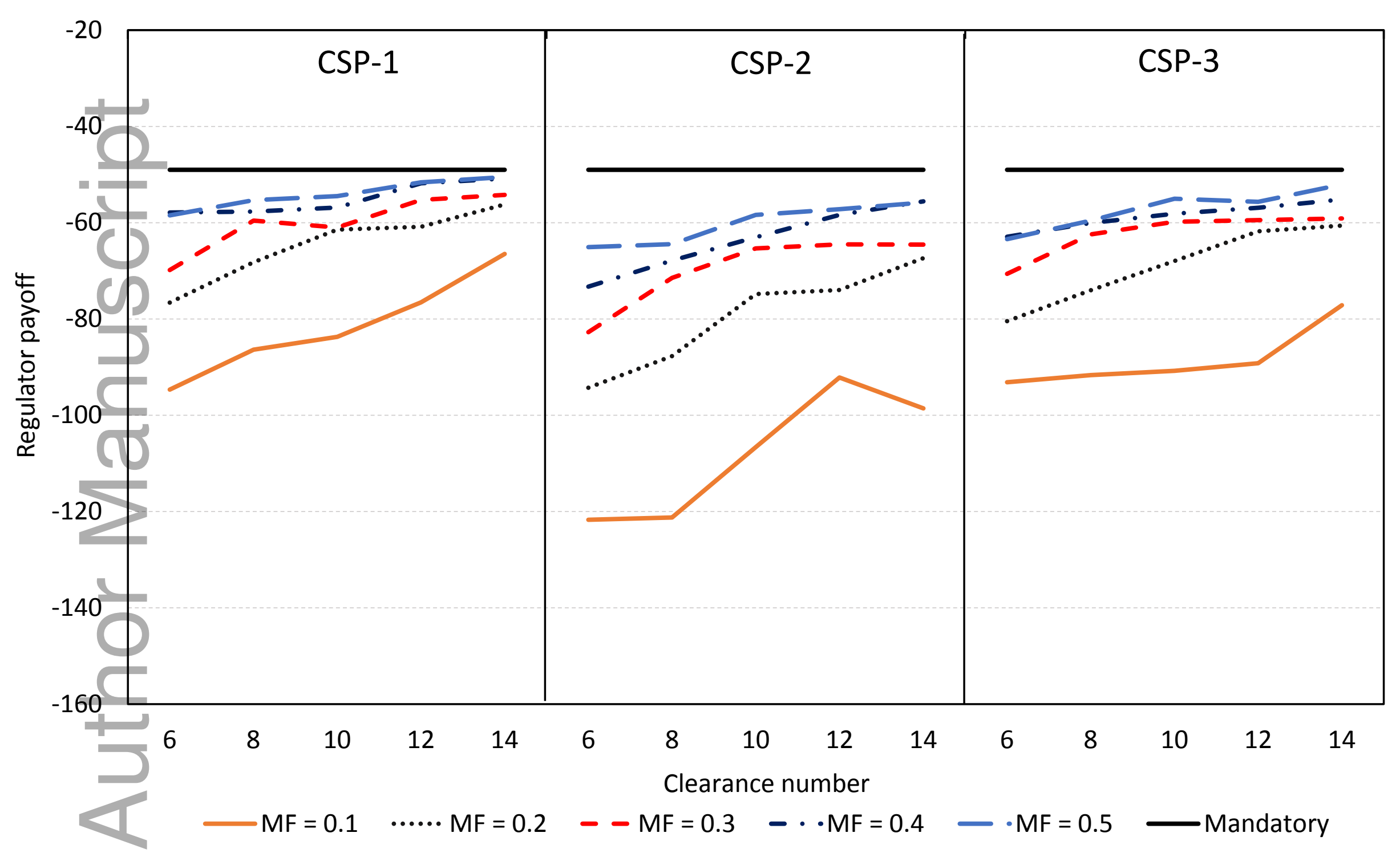

This article is protected by copyright. All rights reserved 


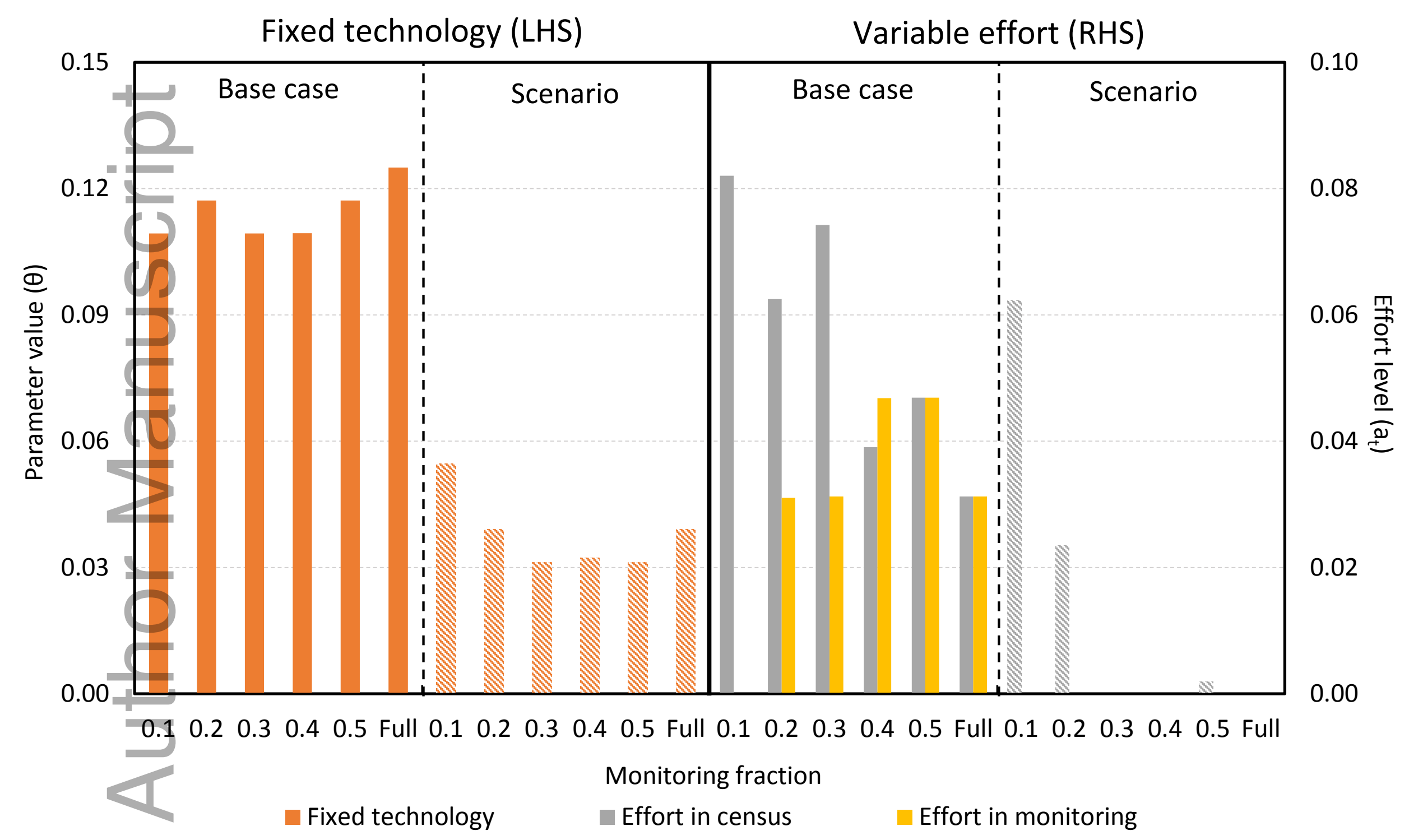




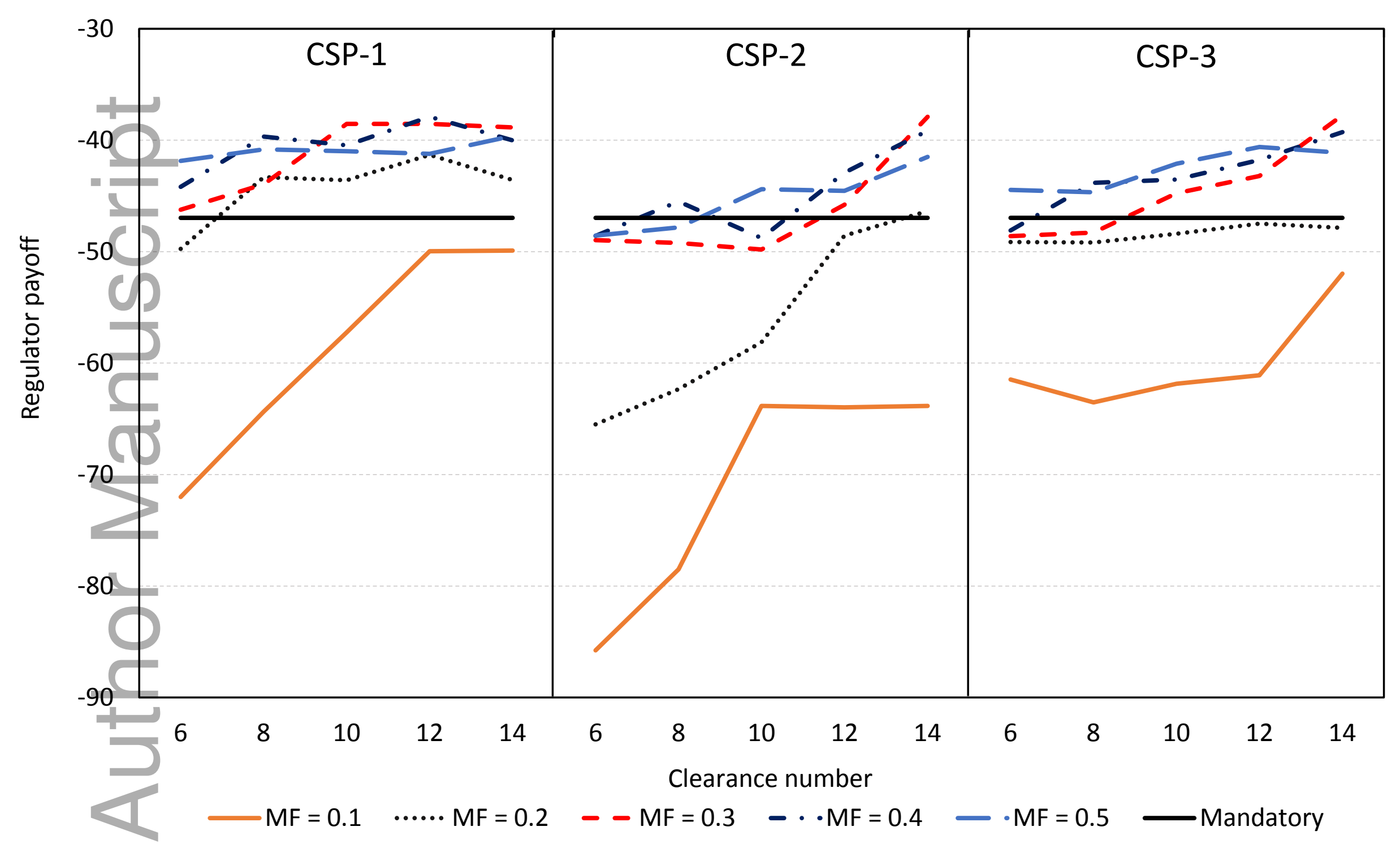

This article is protected by copyright. All rights reserved 


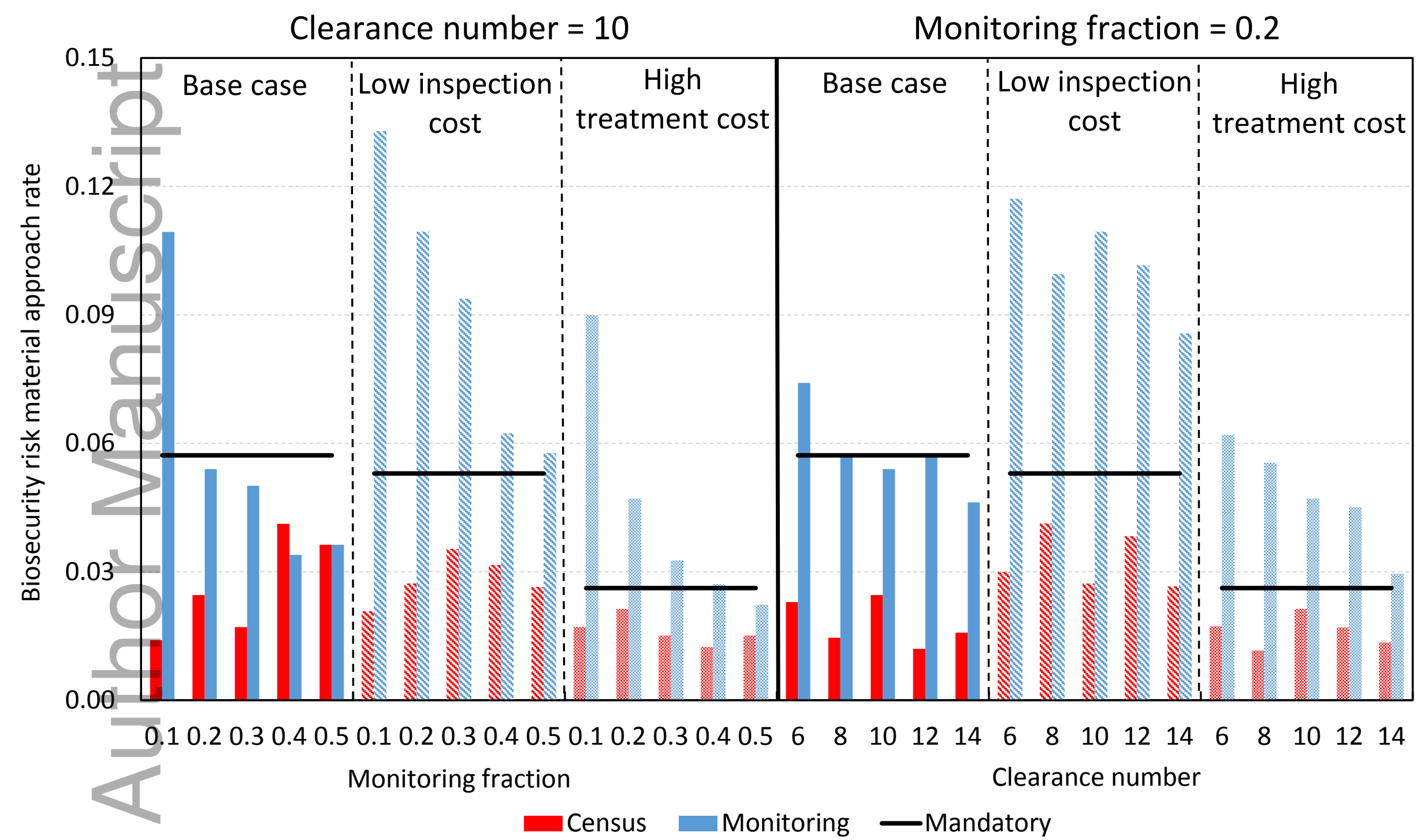




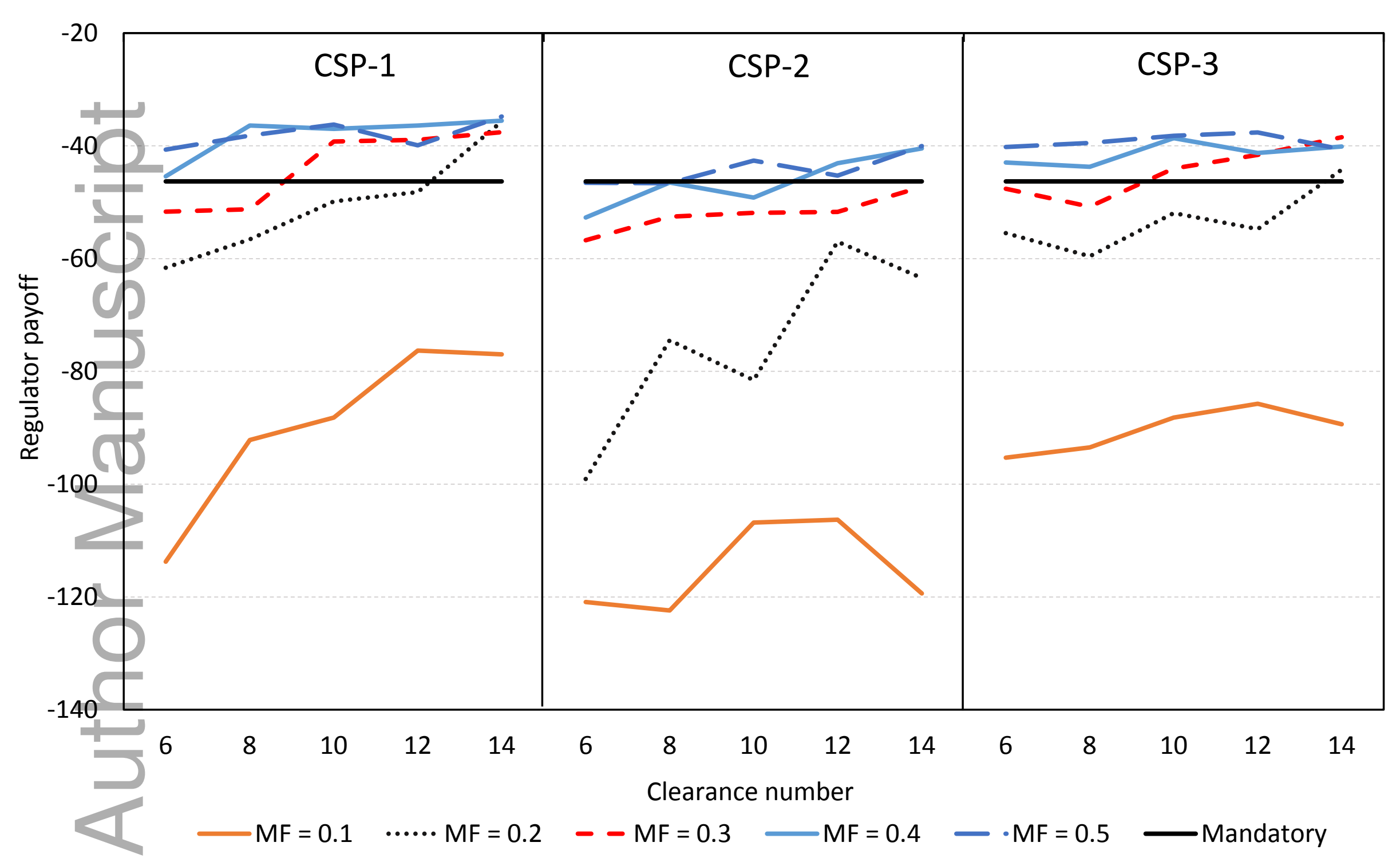

This article is protected by copyright. All rights reserved 


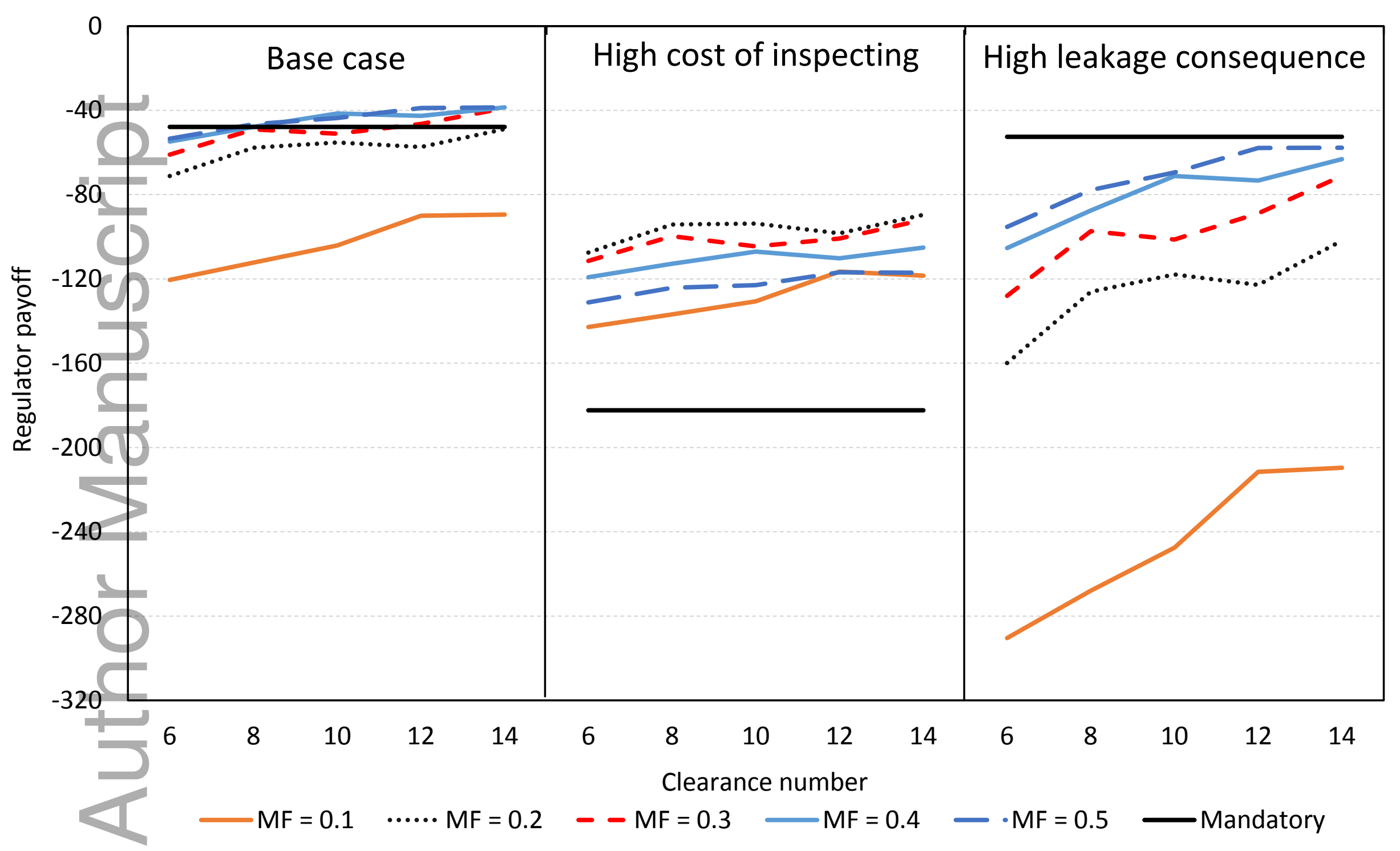

This article is protected by copyright. All rights reserved 


\section{University Library}

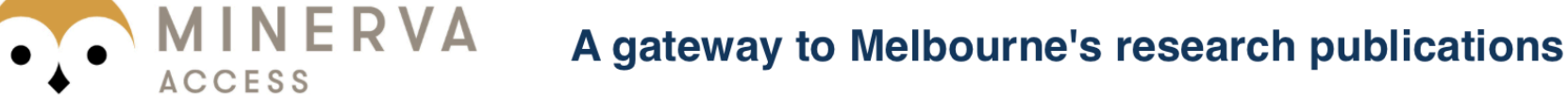

Minerva Access is the Institutional Repository of The University of Melbourne

Author/s:

Rossiter, A;Hester, SM

Title:

Designing Biosecurity Inspection Regimes to Account for Stakeholder Incentives: An Inspection Game Approach

Date:

2017-06-01

Citation:

Rossiter, A. \& Hester, S. M. (2017). Designing Biosecurity Inspection Regimes to Account for Stakeholder Incentives: An Inspection Game Approach. Economic Record, 93 (301), pp.277-301. https://doi.org/10.1111/1475-4932.12315.

Persistent Link:

http://hdl.handle.net/11343/292475 